\title{
Characteristic varieties of quasi-projective manifolds and orbifolds
}

\author{
ENRIQUE ARTAL BARTOLO \\ José IgnACiO Cogolludo-Agustín \\ DANIEL MATEI
}

\begin{abstract}
The present paper considers the structure of the space of characters of quasi-projective manifolds. Such a space is stratified by the cohomology support loci of rank one local systems called characteristic varieties. The classical structure theorem of characteristic varieties is due to Arapura and it exhibits the positive-dimensional irreducible components as pull-backs obtained from morphisms onto complex curves. In this paper a different approach is provided, using morphisms onto orbicurves, which accounts also for zero-dimensional components and gives more precise information on the positive-dimensional characteristic varieties. In the course of proving this orbifold version of Arapura's structure theorem, a gap in his proof is completed. As an illustration of the benefits of the orbifold approach, new obstructions for a group to be the fundamental group of a quasi-projective manifold are obtained.
\end{abstract}

32S20, 32S50, 58K65; 14B05, 14H30, 14H50

\section{Introduction}

The framework of this paper is the study of properties of fundamental groups of complements of hypersurfaces in a projective space, or more generally, of smooth quasi-projective varieties. The approach we take is a classical one, namely to relate cohomological invariants of the variety (or its fundamental group) to its fibrations over a smooth curve, sometimes referred to as pencils. This strong relationship has a long history, going back to Castelnuovo and de Franchis; see Catanese [16]. The cohomological invariants we consider are the jumping loci of twisted cohomology of rank one local systems on the variety. The most general structure theorem for these loci was discovered by Arapura, who described them in terms of fibrations over curves.

We propose here a different approach to obtain another structure theorem, where the base curve of the fibration is viewed as an orbifold. The language of orbifolds allows us to improve Arapura's description, and also to extract finer quasi-projectivity obstructions. Our main goal is to prove the following result. 
Theorem 1 Let $X$ be a smooth quasi-projective variety and let $\Sigma_{k}(X)$ be the $k^{\text {th }}$ characteristic variety of $X$. Let $V$ be an irreducible component of $\Sigma_{k}(X)$. Then one of the two following statements holds.

(1) There exists an orbifold $C_{\varphi}$ supported by a smooth algebraic curve $C$, a surjective orbifold morphism $f: X \rightarrow C_{\varphi}$ and an irreducible component $W$ of $\Sigma_{k}\left(\pi_{1}^{\mathrm{orb}}\left(C_{\varphi}\right)\right)$ such that $V=f^{*}(W)$.

If this is the case we say that $V$ is of pencil type.

(2) $V$ is an isolated torsion point not of pencil type.

The characteristic varieties of a space depend only on its fundamental group and can be seen as a generalization of the Alexander polynomial. They can be defined in terms of jumping loci of the cohomology of local systems. These invariants have been extensively studied from different perspectives. They are closely related to the Green-Lazarsfeld invariants [32] and to the Bieri-Neumann-Strebel invariants [9] of groups and spaces.

In this context, the study of the geometry of smooth quasi-projective varieties in terms of fibrations onto Riemann surfaces has proved to be very fruitful as its widespread use shows; cf the following contributions by Siu [42], Serrano [39], Beauville [8], Catanese [16], Simpson [40], Bauer [7], and Arapura [1].

This paper originated from our attempt to understand Arapura's work. In [1] the following result is stated in Theorem V.1.6.

Theorem (Arapura [1]) Let $V$ be an irreducible component of $\Sigma_{1}(X)$. We then have:

(1) If $\operatorname{dim} V>0$, then there exists a surjective morphism $f: X \rightarrow C$ onto a smooth algebraic curve $C$, and a torsion character $\tau$ such that $V=\tau f^{*}\left(H^{1}\left(C ; \mathbb{C}^{*}\right)\right)$.

(2) If $\operatorname{dim} V=0$, then $V$ is unitary.

This theorem is a consequence of Proposition V.1.4 from [1]. However, the proof of this proposition given by Arapura is not complete. The key technical tool used there is Timmerscheidt's spectral sequence degeneracy result [43, Theorem 5.1] for unitary local systems $\xi$ on $X$. Note that the relevant $E_{1}$-terms are not associated with the divisor $\mathcal{D}$ compactifying $X=\bar{X} \backslash \mathcal{D}$, but rather with the subdivisor $\mathcal{D}^{\xi}$ of $\mathcal{D}$ consisting of those components along which $\xi$ has non-trivial monodromy. The starting assumption of Arapura's proof, that one can just deal with a local system having nontrivial local monodromy about all components of the divisor $\mathcal{D}$ after replacing $X$ by $X^{\xi}=\bar{X} \backslash \mathcal{D}^{\xi}$, cannot in fact be made. Indeed, as it can be seen in Example 1.9 below, the resulting 
local system may no longer be in $\Sigma_{1}\left(X^{\xi}\right)$. Characters that do not ramify along all components of $\mathcal{D}$ have been considered in the context of complements to projective hypersurfaces (cf Artal, Carmona and Cogolludo [2]) and they seem to be essentially different from those ramifying everywhere as can be seen in the Hodge-theoretical characterization provided by Artal, Cogolludo and Libgober [4, Theorem 5.1] where a character is not ramified everywhere iff it is of weight two. Different techniques than those coming from cohomological properties of compact manifolds are hence required in order to deal with this phenomenon.

Our first goal is to fill the gap in Arapura's proof. This is done in Section 5 in the language of orbifold morphisms. Proposition 4.2 ensures the validity of Arapura's statement in [1, Proposition V.1.4], and Theorem 5.1 is an extension of that statement to non-torsion characters. We also point out that Theorem 1 works for any characteristic variety and not only for $\Sigma_{1}$.

The second goal is to describe, in terms of orbifold morphisms, the translated components appearing in Arapura's work; see also [27] for another approach. Several papers in this direction can be found in the literature. In particular, Theorem 1 can be considered as the quasi-projective version of Delzant's Theorem [24].

Orbifolds have also been used for the study of fundamental groups of smooth algebraic varieties in recent works by Corlette and Simpson [22], and Campana [13; 15]. In [24] Delzant proved the exact compact Kähler manifold analogue of Theorem 1, which was extended to compact Kähler orbifolds in [14].

The third goal is to rule out the existence of non-torsion isolated unitary points in the characteristic varieties of quasi-projective manifolds. In the compact projective (resp. Kähler) case this was known from Simpson [41] (resp. Campana [12]). In the non-compact case it was proved by Libgober [37] for quasi-projective manifolds $X$ with $b_{1}(\bar{X})=0$. Dimca in [27] pointed out that this fact may be deduced from Budur's work [11] for general quasi-projective varieties. This can be done with a considerable amount of work; however, we present here a more direct proof of this fact.

The fourth and final goal is to derive properties of the fundamental groups of quasiprojective varieties from Theorem 1, which can be used as effective quasi-projectivity obstructions. As an example, we present here one of the more striking consequences of Theorem 1.

Proposition 6.9 Let $G$ be a quasi-projective group, and let $V_{1}$ and $V_{2}$ be two distinct irreducible components of $\Sigma_{k}(G)$, resp. $\Sigma_{\ell}(G)$. If $\xi \in V_{1} \cap V_{2}$ is a torsion point, then $\xi \in \Sigma_{k+\ell}(G)$. 
This result was used by the authors in [5] to rule out certain families of groups as fundamental groups of quasi-projective manifolds. We collect other properties in Propositions 6.5 and 6.7. In this way, we recover and extend some of the properties found by Dimca [27; 26; 25], Dimca and Maxim [28], and Dimca, Papadima and Suciu [30; 29] using Arapura's Theorem.

This paper was originally planned with another goal in mind: to prove that only components of pencil type in Theorem 1 could exist. This was in part justified by the heuristic fact that, in all the computed examples, only irreducible components of pencil type were found (even the isolated points). One can also give results in this direction in the rational surface case, where characters of orders 2, 3, 4 and 6 are of pencil type for divisors with rational singularities (see [4], Cogolludo and Libgober [19]).

However, the first two authors have recently found an example (see [3]) of a quasiprojective surface whose isolated points of $\Sigma_{1}$ are not of pencil type (Theorem 1).

The paper is organized as follows. In Section 1 notation for quasi-projective varieties is set and some of their properties are discussed. The concept of characteristic variety is introduced in terms of Betti numbers of the 1-cohomology with values in local systems of coefficients. Some ways to compute this cohomology are sketched and applied in Section 2 to compute characteristic varieties of orbifolds; these computations are probably known by specialists but they do not appear explicitly in the literature. For quasi-projective varieties, the main tool for computation comes from Deligne's work [23], which is recalled in Section 3. Also in this section, some technical results are proved. They are mainly contained, one way or another, in the work of Arapura [1], Timmerscheidt [43], and Beauville [8]. The purpose of this is two-fold: on one hand to strengthen the use of the unitary-holomorphic decompositions of a character [1;8]; on the other hand to prepare the ground for a more precise analysis of the Deligne decomposition of the 1-cohomology for local systems of coefficients, which will be considered in Section 5. In Section 4 the Deligne decomposition into holomorphic and anti-holomorphic parts is analyzed for Riemann surfaces as a main ingredient for the general decomposition. In Section 5 this analysis is carried over to higher-dimensional varieties. The main results are stated and proved in Section 5. The key ingredient is Theorem 5.1, which allows us to prove Theorem 1 for $\Sigma_{k}$ for any $k$, since it states that any element of the twisted cohomology for a quasi-projective group (as long as the character is not of torsion type) is obtained as the pull-back of an element of the twisted cohomology of the orbifold. The strategy to prove Theorem 5.1 is to reduce it to the holomorphically pure cohomology classes. To that end, we first investigate in Proposition 4.2 the relation between the anti-holomorphic parts associated with a character and to its conjugate character. Proposition 5.3 is a generalization of [1, Proposition V.1.3] to the orbifold case. The results of Section 3 are then used to apply 
Arapura's method to characters which are non-torsion, not just non-unitary. These improvements allow us to deal with any non-torsion unitary characters using Delzant's approach. The proof of Proposition 5.6 uses Levitt's interpretation [35] of exceptional classes and Simpson's main result in [40] can be applied. A more direct approach using Delzant's way could be done if the result in [40] were generalized to the quasi-projective case. In Section 6, some improvements of the main theorem are discussed for torsion characters. They are not included in the main theorem because the hypotheses are rather technical. This section also includes a number of applications which follow from Theorem 1 and Section 5. Finally, some examples illustrating the properties are shown in Section 7. More examples, applying these techniques, can also be found in [5].

\section{Acknowledgements}

This work is partially supported by the Spanish Ministry of Education MTM201021740-C02-02. The third author is also partially supported by grant CNCSIS PNII-IDEI 1188/2008 and FMI 53/10 (Gobierno de Aragón).

\section{Preliminaries}

Let $X$ be a smooth quasi-projective variety. Using standard Lefschetz-Zariski theory (see Hamm [33]), since the invariants we are interested in only depend on $G:=\pi_{1}(X)$, $X$ will be assumed to be either a complex curve (a Riemann surface) or a complex surface. In any case there exists a smooth compact complex surface (or complex curve) $\bar{X}$ such that $X=\bar{X} \backslash \mathcal{D}$, where $\mathcal{D}$ is a normal crossing divisor. If necessary, additional blow-ups might be performed in order to obtain a more suitable $\bar{X}$, which will be clear from the context.

Characteristic varieties are invariants of finitely presented groups $G$, and they can be computed using any connected topological space $X$ (having the homotopy type of a finite CW-complex) such that $G=\pi_{1}\left(X, x_{0}\right), x_{0} \in X$ as follows. Let us denote $H:=H_{1}(X ; \mathbb{Z})=G / G^{\prime}$. Note that the space of characters on $G$ is a complex torus

$$
\mathbb{T}_{G}:=\operatorname{Hom}\left(G, \mathbb{C}^{*}\right)=\operatorname{Hom}\left(H, \mathbb{C}^{*}\right)=H^{1}\left(X ; \mathbb{C}^{*}\right) .
$$

Given $\xi \in \mathbb{T}_{G}$, the following local system $\mathbb{C}_{\xi}$ of coefficients over $X$ can be constructed. Let $\pi_{\mathrm{ab}}: \tilde{X}_{\mathrm{ab}} \rightarrow X$ be the universal Abelian covering of $X$. The group $H$ acts freely (on the right) on $\tilde{X}_{\mathrm{ab}}$ by the deck transformations of the covering. The local system of coefficients $\mathbb{C}_{\xi}$ is defined as the locally constant sheaf associated with

$$
\pi_{\xi}: \tilde{X}_{\mathrm{ab}} \times_{H} \mathbb{C} \rightarrow X \quad \text { where } \tilde{X}_{\mathrm{ab}} \times_{H} \mathbb{C}:=\left(\tilde{X}_{\mathrm{ab}} \times \mathbb{C}\right) /(x, t) \sim\left(x^{h}, \xi\left(h^{-1}\right) t\right) .
$$


Definition 1.1 The $k^{\text {th }}$ characteristic variety of $G$ is the subvariety of $\mathbb{T}_{G}$ defined by

$$
\Sigma_{k}(G):=\left\{\xi \in \mathbb{T}_{G} \mid \operatorname{dim} H^{1}\left(X, \mathbb{C}_{\xi}\right) \geq k\right\},
$$

where $H^{1}\left(X, \mathbb{C}_{\xi}\right)$ is classically called the twisted cohomology of $X$ with coefficients in the local system $\xi \in \mathbb{T}_{G}$.

It is also customary to use $\Sigma_{k}(X)$ for $\Sigma_{k}(G)$ whenever $\pi_{1}(X)=G$.

1.2 Topological construction of $H^{1}\left(X ; \mathbb{C}_{\xi}\right)$ By duality, we will concentrate our attention on describing the simpler object $H_{1}\left(X ; \mathbb{C}_{\xi}\right)$. Let us suppose that $X$ is a finite $\mathrm{CW}$-complex. Then, $\widetilde{X}_{\mathrm{ab}}$ also inherits a $\mathrm{CW}$-complex structure. Since $H$ is the group of automorphisms of $\pi_{\mathrm{ab}}, H$ acts freely on the set of cells of $\tilde{X}$, and thus the chain complex $C_{*}\left(\tilde{X}_{\mathrm{ab}} ; \mathbb{C}\right)$ becomes a free $\Lambda$-module of finite rank, where $\Lambda:=\mathbb{C}[H]$ is the group algebra of $H$. Given a character $\xi \in \mathbb{T}_{G}, \mathbb{C}$ acquires a $\Lambda$-module structure denoted $\mathbb{C}_{\xi}$ (obtained by the evaluation of $\xi$ on the elements of $H$ ). The twisted chain complex $C_{*}(X ; \mathbb{C})^{\xi}:=C_{*}\left(\tilde{X}_{\mathrm{ab}} ; \mathbb{C}\right) \otimes_{\Lambda} \mathbb{C}_{\xi}$ is, as a vector space, isomorphic to the finite-dimensional complex space $C_{*}(X ; \mathbb{C})$ with twisted differential. This construction implies that the $\Sigma_{k}(G)$ are algebraic subvarieties of $\mathbb{T}_{G}$ defined over $\mathbb{Q}$.

Moreover,

$$
\Sigma_{k}(G) \backslash \mathbf{1}=\operatorname{Char}_{k}\left(H^{1}\left(\tilde{X}_{\mathrm{ab}}\right)\right) \backslash \mathbf{1}=\operatorname{Char}_{k+1}\left(\tilde{H}^{1}\left(\tilde{X}_{\mathrm{ab}}\right)\right) \backslash \mathbf{1}
$$

(cf Cogolludo [18, Section 1.2] or Hironaka [34]), where, if $M$ is a finitely generated $\Lambda$-module then $\operatorname{Char}_{k}(M)$ is the algebraic variety associated with the annihilator of the module $\wedge^{k} M$. Finally, a presentation matrix for the module $\tilde{H}^{1}\left(C_{*}(X ; \mathbb{C})^{\xi}\right)$ is given by evaluation of the Fox matrix of $G$ via the character $\xi$. This matrix is obtained using Fox calculus [31] and it will be extensively used in Section 2.

Example 1.3 Let $G:=\left\langle x_{1}, \ldots, x_{n} \mid R_{1}, \ldots, R_{s}\right\rangle$ be a presentation of $G$ and let $K$ be the CW-complex associated with the presentation. This $\mathrm{CW}$-complex has one 0 -cell, say $P, n 1$-cells denoted by $x_{1}, \ldots, x_{n}$ such that the 1 -skeleton is a wedge of $n$ circles, and $s$ 2-cells $R_{1}, \ldots, R_{S}$ such that their attachments to the 1 -skeleton are determined by the corresponding words. Let $\xi: G \rightarrow \mathbb{C}^{*}$ be a character and let us denote $t_{i}:=\xi\left(x_{i}\right)$. Then the twisted differential of the complex $C_{*}(K ; \mathbb{C})^{\xi}$ is defined as follows:

- $\partial_{1}\left(x_{i}\right)=\left(t_{i}-1\right) P$

- The map $\partial_{2}$ is determined by a map $\varphi$ defined on the free group in $x_{1}, \ldots, x_{n}$, which is defined inductively:

- The image of the empty word under $\varphi$ is 0 . 
- For a word $w$, we have:

$$
\begin{aligned}
& * \varphi\left(x_{i} w\right):=x_{i}+t_{i} \varphi(w) \\
& * \varphi\left(x_{i}^{-1} w\right):=-t_{i}^{-1} x_{i}+t_{i}^{-1} \varphi(w)
\end{aligned}
$$

Note that $\varphi\left(x_{i}^{k} w\right)=\frac{t_{i}^{k}-1}{t_{i}-1} x_{i}+t_{i}^{k} \varphi(w)$.

1.4 Algebraic construction of $H^{1}\left(X ; \mathbb{C}_{\xi}\right)$ There is another way to compute the cohomology $H^{1}\left(X ; \mathbb{C}_{\xi}\right)=H^{1}\left(G ; \mathbb{C}_{\xi}\right)$, which can be seen as the quotient of cocycles by coboundaries. A cocycle is a map $\alpha: G \rightarrow \mathbb{C}$ such that $\alpha(g h)=\alpha(g)+\xi(g) \alpha(h)$. Coboundaries are generated by the mapping $g \mapsto \xi(g)-1$. A cocycle defines a representation $G \rightarrow \operatorname{GL}(2 ; \mathbb{C}), g \mapsto\left(\begin{array}{cc}\xi(g) \alpha(g) \\ 0 & 1\end{array}\right)$. Note that the coboundary representation is reducible.

Remark 1.5 Let $r:=\operatorname{Rank} H$ and let $\operatorname{Tors}_{G}$ be the torsion subgroup of $H=G / G^{\prime}$. Then $\mathbb{T}_{G}$ is an Abelian complex Lie group with $\mid$ Tors $_{G} \mid$ connected components (each one isomorphic to $\left.\left(\mathbb{C}^{*}\right)^{r}\right)$ satisfying the exact sequence

$$
1 \rightarrow \mathbb{T}_{G}^{\mathbf{1}} \rightarrow \mathbb{T}_{G} \rightarrow \operatorname{Hom}\left(\operatorname{Tors}_{G}, \mathbb{C}^{*}\right) \rightarrow 1,
$$

where $\mathbb{T}_{G}^{\mathbf{1}}:=\operatorname{Hom}\left(H / \operatorname{Tors}_{G}, \mathbb{C}^{*}\right)$ is the connected component containing the trivial character $\mathbf{1}$, which is isomorphic to $\left(\mathbb{C}^{*}\right)^{r}$ via the choice of a basis of the lattice $H / \operatorname{Tors}_{G}$. Given $\rho \in \operatorname{Hom}\left(\operatorname{Tors}_{G}, \mathbb{C}^{*}\right)$, we will refer to the component of $\mathbb{T}_{G}$ whose image is $\rho$ as $\mathbb{T}_{G}^{\rho}$. Since the exact sequence (1-2) splits, the elements $\rho$ can be considered to be in $\mathbb{T}_{G}$, and $\mathbb{T}_{G}^{\rho}$ can also be thought of as the only connected component of $\mathbb{T}_{G}$ passing through $\rho$.

When $X$ is a quasi-projective (or Kähler) manifold, the work of Deligne [23] gives a way to compute the twisted cohomology in terms of geometric properties. Let us give some details about this computation. Fix a projective manifold $\bar{X}$ such that $\mathcal{D}:=\bar{X} \backslash X$ is a normal crossing divisor.

Definition 1.6 Let $D$ be an irreducible component of $\mathcal{D}$ and let $\xi \in H^{1}\left(X ; \mathbb{C}^{*}\right)$. We say that $\xi$ does not ramify, or has trivial monodromy, along $D$ if $\xi\left(\mu_{D}\right)=1$, where $\mu_{D}$ is a meridian of $D$. Otherwise, we say that $\xi$ ramifies along $D$.

Remark 1.7 By a meridian of $D$, we simply mean the boundary of a sufficiently small disk intersecting $D$ transversally and only at one point. This means nothing but the boundary of a fiber of the tubular neighborhood (seen as sub-bundle of the normal fibered bundle) on the smooth part of $D$. Since the tubular neighborhood of the smooth part of $D$ in $\mathcal{D}$ is connected, all meridians of $D$ are homologically equivalent. 
Remark 1.8 Let $\mathcal{D}^{\xi}$ be the subdivisor of $\mathcal{D}$ formed by the irreducible components $D$ of $\mathcal{D}$ such that $\xi$ ramifies along $D$. Let $X^{\xi}:=\bar{X} \backslash \mathcal{D}^{\xi}$ and $G^{\xi}:=\pi_{1}\left(X^{\xi}\right)$. Despite the notation, $X^{\xi}$ and $G^{\xi}$ depend on $\bar{X}$. Note that $\xi$ naturally determines an element $\xi_{0} \in \mathbb{T}_{G \xi}=H^{1}\left(X^{\xi} ; \mathbb{C}^{*}\right)$. It is clear that $\xi_{0} \in \Sigma_{1}\left(X^{\xi}\right)$ implies that $\xi \in \Sigma_{1}(X)$, but note that the converse is not true in general as the following example shows. This stresses a common as well as subtle misconception had when trying to study characters on $X$. Therefore, one cannot assume, changing $X$ by $X^{\xi}$, that a character $\xi$ ramifies along all irreducible components of $\mathcal{D}$.

Example 1.9 Let $X:=\mathbb{P}^{1} \backslash\{0,1, \infty\}$. The group $G:=\pi_{1}(X)$ is free of rank 2 (generated, eg, by meridians of 0 and $\infty$ ). The torus $\mathbb{T}_{G}$ is identified with $\left(\mathbb{C}^{*}\right)^{2}$ via the images of those meridians. It is not hard to prove that $\Sigma_{1}(X)=\mathbb{T}_{G}$; see Proposition 2.10. Let us consider a character $\xi$ defined by $\left(z, z^{-1}\right), z \in \mathbb{C} \backslash\{0,1\}$. In this case, the divisor $\mathcal{D}$ is the set $\{0,1, \infty\}$ but the divisor $\mathcal{D}^{\xi}$ is $\{0, \infty\}$ since $\xi$ does not ramify at 1 . Note that $X^{\xi}=\mathbb{C}^{*}$, whose fundamental group is Abelian. Since $\xi_{0}$ is a non-trivial character, $\xi_{0} \notin \Sigma_{1}\left(X^{\xi}\right)$.

\section{Orbifold groups and characteristic varieties}

Definition 2.1 An orbifold $X_{\varphi}$ is a quasi-projective Riemann surface $X$ with a function $\varphi: X \rightarrow \mathbb{N}$ taking the value 1 outside a finite number of points.

We may think of a neighborhood of a point $P \in X_{\varphi}$ with $\varphi(P)$ as the quotient of a disk (centered at $P$ ) by a rotation of angle $2 \pi / n$. A loop around $P$ is considered to be trivial in $X_{\varphi}$ if its lifting bounds a disk. Following this idea, orbifold fundamental groups can be defined as follows.

Definition 2.2 For an orbifold $X_{\varphi}$, let $p_{1}, \ldots, p_{n} \in X$ be the points such that $m_{j}:=$ $\varphi\left(p_{j}\right)>1$. Then, the orbifold fundamental group of $X_{\varphi}$ is defined as

$$
\pi_{1}^{\text {orb }}\left(X_{\varphi}\right):=\pi_{1}\left(X \backslash\left\{p_{1}, \ldots, p_{n}\right\}\right) /\left\langle\mu_{j}^{m_{j}}=1\right\rangle,
$$

where $\mu_{j}$ is a meridian of $p_{j}$. For simplicity, $X_{\varphi}$ might also be denoted by $X_{m_{1}, \ldots, m_{n}}$ or $X_{\bar{m}}$.

Example 2.3 If $X$ is a compact surface of genus $g$ and $\bar{m}=\left(m_{1}, \ldots, m_{n}\right)$, then

$$
\begin{aligned}
\mathbb{G}_{\bar{m}}^{g} & :=\pi_{1}^{\mathrm{orb}}\left(X_{\bar{m}}\right) \\
& =\left\langle a_{1}, \ldots, a_{g}, b_{1}, \ldots, b_{g}, \mu_{1}, \ldots, \mu_{n} \mid \prod_{i=1}^{g}\left[a_{i}, b_{i}\right]=\prod_{j=1}^{n} \mu_{j}, \mu_{j=1, \ldots, n}^{m_{j}}=1\right\rangle .
\end{aligned}
$$


If $X$ is not compact and $\pi_{1}(X)$ is free of rank $r$, then

$$
\mathbb{F}_{\bar{m}}^{r}:=\pi_{1}^{\mathrm{orb}}\left(X_{\bar{m}}\right)=\left\langle a_{1}, \ldots, a_{r}, \mu_{1}, \ldots, \mu_{n}\left|\begin{array}{c}
\mu_{j}^{m_{j}}=1 \\
j=1, \ldots, n
\end{array}\right| .\right.
$$

Definition 2.4 Let $X_{\varphi}$ be an orbifold and $Y$ a smooth algebraic variety. A dominant algebraic morphism $f: Y \rightarrow X$ defines an orbifold morphism $Y \rightarrow X_{\varphi}$ if for all $p \in X$, the divisor $f^{*}(p)$ is a $\varphi(p)$-multiple. The orbifold $X_{\varphi}$ is said to be maximal (with respect to $f$ ) if no divisor $f^{*}(p)$ is an $n$-multiple for $n>\varphi(p)$.

Remark 2.5 If $Y$ is a smooth algebraic variety, $X$ is a quasi-projective Riemann surface and $f: Y \rightarrow X$ is a dominant algebraic morphism, it is possible to define an orbifold structure $\varphi: X \rightarrow \mathbb{N}$, where if $p \in X, \varphi(p)$ is the gcd of the multiplicities of the irreducible components of the divisor $f^{*}(p)$. This structure is maximal if and only if $f$ is surjective.

The following result is well-known. Proofs can be found in [3] or Catanese, Keum and Oguiso [17].

Proposition 2.6 Let $f: Y \rightarrow X$ define an orbifold morphism $Y \rightarrow X_{\varphi}$. Then $f$ induces a morphism $f_{*}: \pi_{1}(Y) \rightarrow \pi_{1}^{\mathrm{orb}}\left(X_{\varphi}\right)$. Moreover, if the generic fiber is connected, then $f_{*}$ is surjective.

Next we compute $\mathbb{T}_{\Pi}$ for orbifold groups $\Pi$.

Proposition 2.7 If $\Pi=\mathbb{F}_{\bar{m}}^{r}$, then $\mathbb{T}_{\Pi}$ is given by the following short exact sequence:

$$
1 \rightarrow \mathbb{T}_{\Pi}^{\mathbf{1}}=\left(\mathbb{C}^{*}\right)^{r} \rightarrow \mathbb{T}_{\Pi} \rightarrow \bigoplus_{j=1}^{n} C_{m_{j}} \rightarrow 1,
$$

(see Remark 1.5) where $C_{m}$ is the cyclic multiplicative group of $m$-roots of unity.

If $\Pi=\mathbb{G}_{\bar{m}}^{g}$, then $\mathbb{T}_{\Pi}$ is given by a similar short exact sequence where the first term is $\mathbb{T}_{\Pi}^{\mathbf{1}}=\left(\mathbb{C}^{*}\right)^{2 g}$ and the last term is the cokernel of the natural mapping $C_{m} \rightarrow \bigoplus_{j=1}^{n} C_{m_{j}}$ where $m:=\operatorname{lcm}\left\{m_{1}, \ldots, m_{n}\right\}$.

Proof This is immediate from the fact that $H=\mathbb{Z}^{r} \oplus C_{m_{1}} \oplus \cdots \oplus C_{m_{n}}$ in the first case and

$$
H=\mathbb{Z}^{2 g} \oplus \frac{C_{m_{1}} \oplus \cdots \oplus C_{m_{n}}}{C_{m}}
$$

in the second case. 
Therefore, for orbifolds coming from Riemann surfaces, the components of $\mathbb{T}_{\Pi}$ are parametrized by the $n$-tuples $\lambda=\left(\lambda_{1}, \ldots, \lambda_{n}\right)$ of roots of unity $\lambda_{j}$ of order $m_{j}$ (resp. whose product is 1 ) if $X$ is non-compact (resp. compact). Let $\mathbb{T}_{\Pi}^{\lambda}$ denote the component of $\mathbb{T}_{\Pi}$ determined by $\lambda$.

Definition 2.8 The number $\ell(\lambda)$ of non-trivial coordinates of $\lambda$ is called the length of the component $\mathbb{T}_{\Pi}^{\lambda}$ of $\mathbb{T}_{\Pi}$. If $\xi \in \mathbb{T}_{\Pi}^{\lambda}$, then this number is also called the length of $\xi$ and it is denoted by $\ell(\xi)$.

Note that there are components of any length $\ell, 0 \leq \ell \leq n$ for $\mathbb{F}_{\bar{m}}^{r}$, whereas this is not the case for $\mathbb{G}_{\bar{m}}^{g}$. The arithmetic of $m_{1}, \ldots, m_{n}$ imposes some conditions; for example, $\mathbb{T}_{\mathbb{G}_{\frac{m}{m}}^{g}}$ cannot have components of length 1 , and if the $m_{i}$ are pairwise coprime, then $\mathbb{T}_{\mathbb{G}_{m}^{g}}^{g}$ cannot have components of length 2 .

Definition 2.9 We define the $k^{\text {th }}$ characteristic variety $\Sigma_{k}\left(X_{\varphi}\right)$ of the orbifold $X_{\varphi}$ as the $k^{\text {th }}$ characteristic variety $\Sigma_{k}(\Pi)$ of its orbifold fundamental group.

Also, if $\xi \in \mathbb{T}_{\Pi}$ is a character on $\Pi$, then $H^{1}\left(X_{\varphi} ; \mathbb{C}_{\xi}\right)$ will denote $H^{1}\left(\Pi ; \mathbb{C}_{\xi}\right)$.

We now compute $\Sigma_{k}\left(X_{\varphi}\right)$ for orbifolds $X_{\varphi}$. In order to do so, we will follow Example 1.3 by considering the CW-complex $K$ associated with the presentation of $\Pi:=\pi_{1}^{\text {orb }}\left(X_{\varphi}\right)$ given in Example 2.3. First we consider the case $\Pi:=\mathbb{F}_{\bar{m}}^{r}$.

Proposition 2.10 Let us consider the group $\Pi:=\pi_{1}^{\mathrm{orb}}\left(X_{\varphi}\right)=\mathbb{F}_{\bar{m}}^{r}$. Then,

$$
\Sigma_{k}\left(X_{\varphi}\right)= \begin{cases}\mathbb{T}_{\Pi} & \text { if } 1 \leq k \leq r-1, \\ \{\mathbf{1}\} \cup \bigcup\left\{\mathbb{T}_{\Pi}^{\lambda} \mid \ell(\lambda) \geq 1\right\} & \text { if } k=r, \\ \bigcup\left\{\mathbb{T}_{\Pi}^{\lambda} \mid \ell(\lambda) \geq k-r+1\right\} & \text { if } r+1 \leq k \leq r+n-1, \\ \varnothing & \text { if } k \geq r+n,\end{cases}
$$

is a decomposition in irreducible components of $\Sigma_{k}\left(X_{\varphi}\right)$.

Proof First let us consider the case where $\xi \neq 1$. Let us consider the complex $C_{*}(K ; \mathbb{C})^{\xi}$; since $\xi \neq \mathbf{1}$, then $\operatorname{dim} \operatorname{ker} \partial_{1}^{\xi}=n+r-1$. The matrix $M$ for $\partial_{2}^{\xi}$ is obtained using Fox calculus and evaluation by $\xi$. Let $\lambda:=\left(\lambda_{1}, \ldots, \lambda_{n}\right)$ the $n$-tuple of roots of unity determining the irreducible component of $\mathbb{T}_{\Pi}$ containing $\xi$. It is easily 
seen that

$$
M:=\left(\begin{array}{cccc}
\frac{\lambda_{1}^{m_{1}}-1}{\lambda_{1}-1} & 0 & \cdots & 0 \\
0 & \frac{\lambda_{2}^{m_{1}}-1}{\lambda_{2}-1} & \cdots & 0 \\
\vdots & \vdots & \ddots & \vdots \\
0 & 0 & \cdots & \frac{\lambda_{n}^{m_{n}}-1}{\lambda_{n}-1} \\
0 & 0 & \cdots & 0 \\
\vdots & \vdots & \ddots & \vdots \\
0 & 0 & \cdots & 0
\end{array}\right) \in M((n+r) \times n, \mathbb{C})
$$

since $\operatorname{Rank} M=n-\ell(\lambda)$, we obtain that $\operatorname{dim} H^{1}\left(K ; \mathbb{C}_{\xi}\right)=r+\ell(\lambda)-1$. On the other side, $\operatorname{dim} H^{1}\left(K ; \mathbb{C}_{1}\right)=\operatorname{Rank} H_{1}(\Pi)=r$.

Now we consider the case $\Pi:=\mathbb{G}_{\bar{m}}^{g}, 2 g+n \geq 2\left(\mathbb{G}_{\bar{m}}^{g}\right.$ is trivial if $\left.2 g+n<2\right)$. The following corrects a mistake in Delzant's statement [24, Proposition 4].

Proposition 2.11 Let us consider the group $\Pi:=\pi_{1}^{\text {orb }}\left(X_{\varphi}\right)=\mathbb{G}_{\bar{m}}^{g}, 2 g+n \geq 2$. Then,

$$
\Sigma_{k}\left(X_{\varphi}\right)= \begin{cases}\mathbb{T}_{\Pi} & \text { if } 1 \leq k \leq 2 g-2, \\ \{\mathbf{1}\} \cup \bigcup\left\{\mathbb{T}_{\Pi}^{\lambda} \mid \ell(\lambda) \geq 2\right\} & \text { if } 2 g-1 \leq k \leq 2 g, \\ \bigcup\left\{\mathbb{T}_{\Pi}^{\lambda} \mid \ell(\lambda) \geq k-2 g+2\right\} & \text { if } 2 g+1 \leq k \leq 2 g+n-2, \\ \varnothing & \text { if } k \geq 2 g+n-1,\end{cases}
$$

is a decomposition in irreducible components of $\Sigma_{k}\left(X_{\varphi}\right)$.

Proof If $\xi=\mathbf{1}, \operatorname{dim} H^{1}\left(K ; \mathbb{C}_{\mathbf{1}}\right)=\operatorname{Rank} H_{1}(\Pi)=2 g$. Let us assume $\xi \neq \mathbf{1}$ and we assume the notation of Example 2.3 and Proposition 2.10: $\lambda=\left(\lambda_{1}, \ldots, \lambda_{n}\right)$ where $\lambda_{i}:=$ $\xi\left(\mu_{i}\right)$; we denote also $x_{i}:=\xi\left(a_{i}\right)$ and $y_{i}:=\xi\left(b_{i}\right)$. We have dim ker $\partial_{1}^{\xi}=2 g+n-1$ 
and we obtain the matrix $M$ for $\partial_{2}^{\xi}$ using Fox calculus and evaluation by $\xi$ :

$M:=\left(\begin{array}{ccccc}\frac{\lambda_{1}^{m_{1}}-1}{\lambda_{1}-1} & 0 & \cdots & 0 & 1 \\ 0 & \frac{\lambda_{2}^{m_{1}}-1}{\lambda_{2}-1} & \cdots & 0 & \lambda_{1} \\ \vdots & \vdots & \vdots & 0 & \vdots \\ 0 & 0 & \cdots & \frac{\lambda_{n}^{m_{n}}-1}{\lambda_{n}-1} & \lambda_{1} \cdots \lambda_{n-1} \\ & & & & \\ 0 & 0 & \cdots & 0 & y_{1}-1 \\ 0 & 0 & \cdots & 0 & 1-x_{1} \\ 0 & \vdots & \ddots & \vdots & \vdots \\ 0 & 0 & \cdots & 0 & y_{g}-1 \\ 0 & 0 & 1-x_{g}\end{array}\right) \in M((n+2 g) \times(n+1), \mathbb{C})$

Since Rank $M=n+1-\ell(\lambda)$, we obtain that $\operatorname{dim} H^{1}\left(K ; \mathbb{C}_{\xi}\right)=2 g+\ell(\lambda)-2$. The result follows since the case $\ell(\lambda)=1$ cannot arise.

As an immediate corollary of Propositions 2.10 and 2.11, the twisted cohomologies of a character, its inverse, and its conjugate can be related as follows.

Proposition 2.12 Let $X_{\varphi}$ be an orbifold, let $\Pi:=\pi_{1}^{\text {orb }}\left(X_{\varphi}\right)$, and let $\xi \in \mathbb{T}_{\Pi}$. Then

$$
\operatorname{dim} H^{1}\left(\Pi ; \mathbb{C}_{\xi}\right)=\operatorname{dim} H^{1}\left(\Pi ; \mathbb{C}_{\xi^{-1}}\right)=\operatorname{dim} H^{1}\left(\Pi ; \mathbb{C}_{\bar{\xi}}\right) .
$$

Proof In the proofs of Propositions 2.10 and 2.11 it was computed that

$$
\operatorname{dim} H^{1}\left(\Pi ; \mathbb{C}_{\xi}\right)= \begin{cases}\operatorname{Rank} H_{1}(\Pi) & \text { if } \xi=\mathbf{1}, \\ \ell(\lambda)-\chi(X) & \text { if } \xi \in \mathbb{T}_{\Pi}^{\lambda}\end{cases}
$$

Clearly, if $\xi \in \mathbb{T}_{\Pi}^{\lambda}$ then $\xi^{-1} \in \mathbb{T}_{\Pi}^{\lambda^{-1}}$ and $\bar{\xi} \in \mathbb{T}_{\Pi}^{\bar{\lambda}}$. Moreover $\ell(\lambda)=\ell\left(\lambda^{-1}\right)=\ell(\bar{\lambda})$, and the statement follows.

As a consequence of Propositions 2.10 and 2.11 the twisted cohomology of an orbifold $X_{\varphi}$ can be identified with the twisted cohomology of a Riemann surface.

Proposition 2.13 Let $X_{\varphi}$ be an orbifold with singular points $p_{1}, \ldots, p_{n}$ and orbifold fundamental group $\Pi:=\pi_{1}^{\text {orb }}\left(X_{\varphi}\right)$. For $\xi \in \mathbb{T}_{\Pi}$, set $Y:=X \backslash\left\{p_{j} \in X \mid \xi\left(\mu_{j}\right) \neq 1\right\}$. Let $\xi_{1}$ be the character on $Y$ determined by $\xi$. Then $H^{1}\left(X_{\varphi} ; \mathbb{C}_{\xi}\right)$ is naturally identified with $H^{1}\left(Y ; \mathbb{C}_{\xi_{1}}\right)$. 
Proof The particular cases of Propositions 2.10 and 2.11 where no orbifold points are present give the dimensions of the twisted cohomology groups of a Riemann surface $Y$ :

$$
\operatorname{dim} H^{1}\left(Y ; \mathbb{C}_{\xi}\right)= \begin{cases}\operatorname{Rank} H_{1}(Y) & \text { if } \xi=\mathbf{1}, \\ -\chi(Y) & \text { if } \xi \neq \mathbf{1}\end{cases}
$$

Now fix $J$ a subset of $\{1, \ldots, n\}$ of size $l$ and suppose $\xi$ is such that $\xi\left(\mu_{j}\right) \neq 1$ precisely when $j \in J$. Then $\xi \in \mathbb{T}_{\Pi}^{\lambda}$ and $\ell(\lambda)=\ell$. We then have that

$$
\operatorname{dim} H^{1}\left(X_{\varphi} ; \mathbb{C}_{\xi}\right)= \begin{cases}\operatorname{Rank} H_{1}(\Pi) & \text { if } \xi=\mathbf{1}, \\ \ell-\chi(X) & \text { if } \xi \neq \mathbf{1} .\end{cases}
$$

Clearly $Y=X \backslash\left\{p_{j} \mid j \in J\right\}$ is a Riemann surface of Euler characteristic $\chi(Y)=$ $\chi(X)-\ell$ and first Betti number Rank $H_{1}(Y)=\operatorname{Rank} H_{1}(X)+\ell=\operatorname{Rank} H_{1}(\Pi)+\ell$. It follows that $\operatorname{dim} H^{1}\left(Y ; \mathbb{C}_{\xi}\right)=\operatorname{dim} H^{1}\left(X_{\varphi} ; \mathbb{C}_{\xi}\right)$, and so the restriction $H^{1}\left(Y ; \mathbb{C}_{\xi}\right) \rightarrow$ $H^{1}\left(X_{\varphi} ; \mathbb{C}_{\xi}\right)$ is an isomorphism.

\section{Deligne's theory and Hodge-like decompositions}

In what follows, we briefly summarize Deligne's results [23] (with some addenda by Timmerscheidt [43]) on twisted cohomology of quasi-projective varieties. Let $\xi \in \mathbb{T}_{G}$, $G:=\pi_{1}(X)$. Consider the line bundle $L_{\xi}:=\mathbb{C}_{\xi} \otimes \mathcal{O}_{X}$ over $X$. The local system of coefficients $\mathbb{C}_{\xi}$ induces a flat connection $\nabla$ on $L_{\xi}$. Let us fix $\bar{L}_{\xi}$ an extension of $L_{\xi}$ to $\bar{X}$, whose associated flat connection $\bar{\nabla}$ is meromorphic (having $\log$ poles along $\mathcal{D}$ ) and extends $\nabla$. Note that plenty of such extensions are possible (there is a choice of a logarithm determination around every component $D$ of $\mathcal{D}$ ). More precisely, fix an irreducible component $D$ of $\mathcal{D}$ and let $p \in D \backslash \operatorname{Sing}(\mathcal{D})$. Let $u, v$ be a local analytical system of coordinates centered at $p$ such that $v=0$ is the local equation of $D$. Let $\mu_{D}$ be a meridian of $D$ and let $\xi\left(\mu_{D}\right)=: t$. The extension $\bar{\nabla}$ to $D$ is determined by the choice of $\alpha \in \mathbb{C}$ such that $\exp (2 \sqrt{-1} \pi \alpha)=t$, or equivalently, such that $v^{\alpha}$ is the equation of a multivalued flat section on a suitable chart of $\bar{L}_{\xi}$.

Definition 3.1 We say that $\alpha$ is the residue of the meromorphic extension $\bar{\nabla}$ around $D$.

Definition 3.2 An extension $\bar{L}_{\xi}$ as above is said to be suitable if the residues of $\bar{L}_{\xi}$ around the components of $\mathcal{D}$ are not positive integers. The Deligne extension of $\left(L_{\xi}, \nabla\right)$ is the unique holomorphic extension of $L_{\xi}$ whose associated meromorphic flat connection (with log poles) is such that its residues around any component of $\mathcal{D}$ have real parts in $[0,1)$. Such an extension will be denoted by $\tilde{L}_{\xi}$. 
The main result proved by Deligne in [23] states that if $\bar{L}_{\xi}$ is suitable then the hypercohomology of the twisted complex of holomorphic sheaves of logarithmic forms with poles along $\mathcal{D}$ (denoted by $\left.\Omega_{\bar{X}}^{\bullet}(\log \mathcal{D}) \otimes \bar{L}_{\xi}\right)$ is isomorphic to the twisted cohomology of $X$ with coefficients in $\xi$, that is,

$$
\mathbb{H}^{i}\left(\bar{X} ; \Omega_{\bar{X}}^{\bullet}(\log \mathcal{D}) \otimes \bar{L}_{\xi}\right) \cong H^{i}\left(X ; \mathbb{C}_{\xi}\right) .
$$

This induces a decomposition $H^{1}\left(X ; \mathbb{C}_{\xi}\right)=H_{\xi}^{\mathcal{O}} \oplus H_{\xi}^{\overline{\mathcal{O}}}$, where $H_{\xi}^{\mathcal{O}}$ corresponds to the $(1,0)$-term and $H_{\xi}^{\overline{\mathcal{O}}}$ corresponds to the $(0,1)$-term. In a nutshell, Timmerscheidt [43] showed that, in the case of Deligne extensions of unitary bundles, the associated spectral sequence degenerates in the first step. Next, we will describe some of the main properties derived from this result, some of which are particular to the Deligne extension.

Theorem 3.3 [23; 43] The following properties hold:

(1) If $\bar{L}_{\xi}$ is a suitable extension, then the space $H_{\xi}^{\mathcal{O}}$ is the homology of the complex

$$
H^{0}\left(\bar{X} ; \bar{L}_{\xi}\right) \stackrel{\bar{\nabla}}{\longrightarrow} H^{0}\left(\bar{X} ; \Omega_{\bar{X}}^{1}(\log \mathcal{D}) \otimes \bar{L}_{\xi}\right) \stackrel{\bar{\nabla}}{\longrightarrow} H^{0}\left(\bar{X} ; \Omega_{\bar{X}}^{2}(\log \mathcal{D}) \otimes \bar{L}_{\xi}\right)
$$

and $H_{\xi}^{\overline{\mathcal{O}}}$ is the kernel of

$$
\bar{\nabla}: H^{1}\left(\bar{X} ; \bar{L}_{\xi}\right) \rightarrow H^{1}\left(\bar{X} ; \Omega_{\bar{X}}^{1}(\log \mathcal{D}) \otimes \bar{L}_{\xi}\right) .
$$

(2) If $\xi$ is unitary and $\widetilde{L}_{\xi}$ is the Deligne extension then $\bar{\nabla}=0$ in (3-2) and (3-3), ie,

$$
H_{\xi}^{\mathcal{O}}=H^{0}\left(\bar{X} ; \Omega_{\bar{X}}^{1}(\log \mathcal{D}) \otimes \widetilde{L}_{\xi}\right), \quad H_{\xi}^{\overline{\mathcal{O}}}=H^{1}\left(\bar{X} ; \widetilde{L}_{\xi}\right)
$$

Remark 3.4 This decomposition is, in general, non-canonical. As in Theorem 3.3(2) more properties can be derived when $\xi$ is unitary. Following the ideas in [1, III-IV], the decomposition $H^{1}\left(X ; \mathbb{C}_{\xi}\right)=H_{\xi}^{\mathcal{O}} \oplus H_{\xi}^{\overline{\mathcal{O}}}$ is natural and carries a mixed Hodge structure.

Definition 3.5 An element $0 \neq \theta \in H^{1}\left(X ; \mathbb{C}_{\xi}\right)$ is said to be holomorphically (resp. anti-holomorphically) pure if $\theta \in H_{\xi}^{\mathcal{O}}$ (resp. $\theta \in H_{\xi}^{\overline{\mathcal{O}}}$ ).

The following is yet another consequence of the Hodge theory on the cohomology of $X$, which will be very useful for our purposes. The statement appears in the proof of [1, Proposition V.1.4] (where $X$ must be replaced by $\bar{X}$ in the last summand). 
Proposition 3.6 There is a natural real decomposition

$$
H^{1}(X ; \mathbb{C})=\left(H^{10}(X) \oplus H_{\mathbb{R}}^{11}(X)\right) \oplus \sqrt{-1}\left(H_{\mathbb{R}}^{11}(X) \oplus H^{1}(\bar{X} ; \mathbb{R})\right) .
$$

The sum of the first three terms corresponds to $H^{0}\left(\bar{X} ; \Omega_{\bar{X}}^{1}(\log \mathcal{D})\right)$, whereas the sum of the first two corresponds to those forms having purely imaginary residues. The residues along the components of $\mathcal{D}$ of the forms in the first and last terms are trivial.

\subsection{Character decompositions $[1 ; 8]$ Let $\xi \in \mathbb{T}_{G}=H^{1}\left(X ; \mathbb{C}^{*}\right)$. Note that} there exists a torsion element $\tau$ such that $\tilde{\xi}:=\tau^{-1} \xi \in \mathbb{T}_{G}^{\mathbf{1}}$ and there exists $\eta \in$ $H^{1}(X ; \mathbb{C})$ such that $\tilde{\xi}=\exp (\eta)$. The element $\eta$ is unique up to sum by an element in $2 \sqrt{-1} \pi H^{1}(X ; \mathbb{Z})$. According to Proposition 3.6, there exist

$$
\omega \in H^{0}\left(\bar{X} ; \Omega_{\bar{X}}^{1}(\log \mathcal{D})\right) \quad \text { and } \quad \delta \in H^{1}(\bar{X} ; \mathbb{R})
$$

such that $\eta=\omega+\sqrt{-1} \delta$. Summarizing, $\xi=\psi \exp (\omega)$, where $\psi:=\tau \exp (\sqrt{-1} \delta)$ is unitary.

Note that any choice of $\omega_{0} \in H_{\mathbb{R}}^{11}(X)$ leads to another decomposition $\xi=\tilde{\psi} \exp (\widetilde{\omega})$ where

$$
\tilde{\omega}:=\omega-\sqrt{-1} \omega_{0} \quad \text { and } \quad \tilde{\psi}:=\tau \exp \left(\sqrt{-1}\left(\delta+\omega_{0}\right)\right) .
$$

Definition 3.8 A decomposition $\xi=\psi \exp (\omega)$ is called a unitary-holomorphic decomposition of $\xi$ if $\psi$ is unitary and $\omega \in H^{0}\left(\bar{X} ; \Omega_{\bar{X}}^{1}(\log \mathcal{D})\right)$. Such a decomposition is called

- integrally unramified if $\mathcal{D}^{\psi}=\mathcal{D}^{\xi}$ and $\omega$ is holomorphic outside $\mathcal{D}^{\xi}$,

- $\quad$ strict if it is integrally unramified and $\omega \notin 2 \sqrt{-1} \pi H^{1}(X ; \mathbb{Z})$.

Remark 3.9 In the definition of integrally unramified, the condition of being holomorphic outside $\mathcal{D}^{\xi}$ is non-void. Let $\xi=\psi \exp (\omega)$ be an integrally unramified decomposition and let $\eta$ be a logarithmic one-form having integral residues around the components of $\mathcal{D}-\mathcal{D}^{\xi}$. The decomposition $\xi=\psi \exp (\omega+\eta)$ is also unitaryholomorphic but not integrally unramified.

Remark 3.10 Let us fix a unitary-holomorphic decomposition $\xi=\psi \exp (\omega)$. We consider the Deligne extension $\widetilde{L}_{\psi}$ associated with $\psi$. This is also an extension for $L_{\xi}$ and the meromorphic connection is $\nabla_{\omega}:=\bar{\nabla}+\wedge \omega$ (see, eg, [1, Section V] or [8]), where $\bar{\nabla}$ is the connection associated with $\psi:$ it is a flat meromorphic connection extending $\nabla$ and its monodromy equals $\xi$ (using the unitary-holomorphic decomposition).

Lemma 3.11 Let $\xi=\psi \exp (\omega)$ be an integrally unramified unitary-holomorphic decomposition. 
(1) Any integral residue of $\nabla_{\omega}$ vanishes (and, in particular, Theorem 3.3(1) can be applied).

(2) The character $\psi$ (resp. the form $\omega$ ) is a restriction of a unitary character (resp. a logarithmic form) defined on $X^{\xi}$.

Proof Let $D$ be an irreducible component of $\mathcal{D}$ where $\xi$ does not ramify. From the definition of integrally unramified we deduce that $\exp (\omega)$ does not ramify along $D$, and hence, the same happens for $\psi$. Therefore (1) and (2) follow.

The proofs of the results of Section 5 are easier for characters admitting a strict unitaryholomorphic decomposition; see Corollary 5.4. The following result shows under which conditions such decompositions exist. The sufficient condition Lemma 3.12(1) is classical and it is well-known in the projective case while the second sufficient condition is original in this context to our knowledge.

Lemma 3.12 In the following cases the character $\xi$ admits a strict unitary-holomorphic decomposition:

(1) $\xi$ is non-unitary

(2) $b_{1}\left(X^{\xi}\right)>b_{1}(\bar{X})$

Proof By Lemma 3.11(2) it is equivalent to find a strict unitary-holomorphic decomposition for the induced character $\xi_{0}$ in $X^{\xi}$. Then, replacing $X$ by $X^{\xi}$, we may assume that $\xi$ ramifies along every irreducible component of $\mathcal{D}$. Consider $\xi=\psi \exp (\omega)$ a unitary-holomorphic decomposition of $\xi$.

Following Arapura [1] one can choose $\omega_{1} \in H^{1}(X ; \mathbb{Z})$ with non-trivial residues along $\mathcal{D}$. Consider $\eta=\alpha+\beta$ a decomposition where $\alpha \in H^{1}(\bar{X} ; \mathbb{R})$ and $\beta \in H_{\mathbb{R}}^{11}(X)$. Note that

$\xi=\psi \exp (\omega) \exp (2 \pi \sqrt{-1} \eta)=\psi \exp (2 \pi \sqrt{-1}(\alpha+t \beta)) \exp (\omega+2 \pi \sqrt{-1}(1-t) \beta)$.

Note that $\psi_{1}:=\psi \exp (2 \pi \sqrt{-1}(\alpha+t \beta))$ has non-trivial residues along $\mathcal{D}$. Eventually replacing $\psi$ by $\psi_{1}$ and $\omega$ by $\omega+2 \pi \sqrt{-1}(1-t) \beta$ one might assume that $\xi=\psi \exp (\omega)$ is an integrally unramified unitary-holomorphic decomposition of $\xi$.

All that is left to check is that this can be done choosing $\omega \neq \equiv 0 \bmod H^{1}(X ; \mathbb{Z})$.

If (1) holds, then $\omega \notin H^{1}(X ; \mathbb{Z})$ (by Proposition 3.6); otherwise $\xi=\psi$ would be a unitary character.

If (2) holds, then Proposition 3.6 implies that $H_{\mathbb{R}}^{11}(X) \neq 0$. A generic choice of $\omega_{0} \in H_{\mathbb{R}}^{11}(X)$ leads to another decomposition where $\psi$ (resp. $\omega$ ) is replaced by $\psi \exp \left(\sqrt{-1} \omega_{0}\right)$ (resp. $\left.\omega-\sqrt{-1} \omega_{0}\right)$ satisfying $\omega \notin H^{1}(X ; \mathbb{Z})$. 


\section{Anti-holomorphic pure factors of twisted cohomology}

In this section we study the relationship between the twisted cohomologies relative to characters $\xi$ and $\xi^{-1}$ taking into account Deligne's theory. In particular, we study the properties of $H_{\xi^{ \pm 1}}^{\mathcal{O}}$ and $H_{\xi^{ \pm 1}}^{\overline{\mathcal{O}}}$ (see (3-1) and paragraph right after for a definition).

Theorem $4.1 \quad[43 ; 1] \quad$ If the character $\xi$ ramifies along each irreducible component of $\mathcal{D}$, then there is a natural inclusion $H_{\xi}^{\overline{\mathcal{O}} \hookrightarrow} \overline{H_{\xi^{-1}}^{\mathcal{O}}}$.

Theorem 4.1 is proved in [43, Theorem 5.1] for unitary characters (where the inclusion is in fact an isomorphism). The second part of the proof of [1, Proposition V.1.4] provides the general result. We can summarize it as follows. For $\xi=\psi \exp (\omega)$, let $\alpha \in H_{\xi}^{\overline{\mathcal{O}}}$, that is, $\alpha \in H^{1}\left(\bar{X}, \widetilde{L}_{\psi}\right)$ and $\alpha \wedge \omega=0$; cf Remark 3.10 and exact sequence in (3-3). Since $\xi$ ramifies along each irreducible component of $\mathcal{D}$, one has $\widetilde{L}_{\psi}^{-1}=\widetilde{L}_{\psi^{-1}} \otimes \mathcal{O}_{\bar{X}}(-\mathcal{D})($ cf $[43$, Theorem 5.2]). Therefore,

$$
\bar{\alpha} \in H^{0}\left(\bar{X}, \Omega_{\bar{X}}^{1}(\log \mathcal{D}) \otimes \widetilde{L}_{\psi^{-1}}\right) .
$$

According to the $L^{2}$ cohomology arguments in the proof of [1, Proposition V.1.4] one has that $\bar{\alpha} \wedge \omega=0$, and if $\widetilde{L}_{\psi}$ is trivial, then $\bar{\alpha}$ is not a multiple of $\omega$. Thus $0 \neq \bar{\alpha} \in H_{\xi^{-1}}^{\mathcal{O}}$ and the statement follows; see (3-2).

Note that this theorem does not deal with arbitrary characters, but only with those that ramify along every irreducible component of $\mathcal{D}$. In the general case one might have to resort to the conjugated character as we will see in what follows. Let $\xi$ be a character and consider as in Section 1 the divisor $\mathcal{D}^{\xi}$ (containing the components of $\mathcal{D}$ where $\xi$ ramifies) and $X^{\xi}:=\bar{X} \backslash \mathcal{D}^{\xi}$. For the sake of clarity we denote by $\xi_{0}$ the character induced by $\xi$ on $X^{\xi}$.

Proposition 4.2 For any character $\xi$ on $X$ there is a natural isomorphism

$$
H_{\widehat{\xi}}^{\overline{\mathcal{O}}} \cong H_{\widehat{\xi}_{0}}^{\overline{\mathcal{O}}}
$$

where $\hat{\xi}_{0}$ is the character induced by $\hat{\xi}$ on $X^{\widehat{\xi}}$ and $\hat{\xi}$ is either $\xi$ or its conjugate $\bar{\xi}$.

In order to prove this proposition we need to recover more information on the Deligne decomposition of the twisted cohomology of a Riemann surface, which has already been computed in Section 2. The proof will be postponed to the end of the section. Note also that Proposition 2.13 allows to extend the concept of decomposition into a holomorphic and an anti-holomorphic part to $H^{1}\left(X_{\varphi} ; \mathbb{C}_{\xi}\right)$ for orbifolds. 
We consider now some computations of meromorphic extensions for the particular case $X:=\mathbb{P}^{1} \backslash\left\{p_{1}, \ldots, p_{n}\right\}, n>0$, ie, $\mathcal{D} \neq \varnothing$ is the reduced divisor supported on $\left\{p_{1}, \ldots, p_{n}\right\}$. The group $G:=\pi_{1}(X)$ is generated by meridians $\mu_{j}, j=1, \ldots, n$. For a suitable choice of these meridians the only relation is $\mu_{1} \cdots \mu_{n}=1$. Let us fix a character $\xi \in \mathbb{T}_{G}$; an extension $\bar{L}_{\xi}$ to $\mathbb{P}^{1}$ of $L_{\xi}:=\mathbb{C}_{\xi} \otimes \mathcal{O}_{X}$ (with a meromorphic extension $\bar{\nabla}$ of the connection of $\left.L_{\xi}\right)$ is determined by the choice of $\alpha_{j} \in \mathbb{C}, j=$ $1, \ldots, n$, such that $\xi\left(\mu_{j}\right)=\exp \left(-2 \sqrt{-1} \pi \alpha_{j}\right)$. Note that $k:=-\sum_{j=1}^{n} \alpha_{j} \in \mathbb{Z}$. From these choices, the line bundle $\bar{L}_{\xi}$ admits a multivalued flat meromorphic section $\sigma$ having complex order $\alpha_{j}$ at $p_{j}$. We deduce from this fact that $\bar{L}_{\xi} \cong \mathcal{O}_{\mathbb{P}^{1}}(-k)$. Using this idea for arbitrary Riemann surfaces we obtain this useful result, which is well-known.

Proposition 4.3 Let $X$ be a quasi-projective Riemann surface with compactification $\bar{X}, n:=\#(\bar{X} \backslash X)$. Let $\xi$ be a character on $X$ and let $\tilde{L}_{\xi}$ be the Deligne extension of $L_{\xi}$ to $\bar{X}$. The following results hold:

(1) If $n=0$, then $\operatorname{deg} \widetilde{L}_{\xi}=0$.

(2) If $n>0$, then $-n<\operatorname{deg} \tilde{L}_{\xi} \leq 0$.

(3) If $n>0$ and $\xi$ is unitary, then $\xi$ is trivial if and only if $\operatorname{deg} \widetilde{L}_{\xi}=0$.

(4) If $n=0$ and $\xi$ is unitary, then $\xi$ is trivial if and only if $\tilde{L}_{\xi} \cong \mathcal{O}_{\bar{X}}$.

(5) $\tilde{L}_{\xi}$ admits non-zero holomorphic sections if and only if $\widetilde{L}_{\xi} \cong \mathcal{O}_{\bar{X}}$.

Let us continue for a moment our discussion above where the Deligne extension $\widetilde{L}_{\xi}$ for the unitary character $\xi$ has been fixed (hence $\alpha_{j} \in \mathbb{R}$ ). If $\xi$ is not the trivial character, then $0<k<n$. Using Theorem 3.3(2), one has $H_{\xi}^{\mathcal{O}}=H^{0}\left(\mathbb{P}^{1} ; \Omega_{\mathbb{P}^{1}}^{1}(\log \mathcal{D}) \otimes \widetilde{L}_{\xi}\right)$. Since

$$
\Omega_{\mathbb{P}^{1}}^{1}(\log \mathcal{D}) \otimes \widetilde{L}_{\xi}=\mathcal{O}_{\mathbb{P}^{1}}(-2+n-k),
$$

one can deduce that $\operatorname{dim} H_{\xi}^{\mathcal{O}}=n-k-1$. Also $H_{\xi}^{\overline{\mathcal{O}}}=H^{1}\left(\mathbb{P}^{1} ; \tilde{L}_{\xi}\right)$; by Serre duality, this space has the same dimension as

$$
H^{0}\left(\mathbb{P}^{1} ; \Omega_{\mathbb{P}^{1}}^{1} \otimes\left(\tilde{L}_{\xi}\right)^{-1}\right) \quad \text { and } \quad \Omega_{\mathbb{P}^{1}}^{1} \otimes\left(\tilde{L}_{\xi}\right)^{-1}=\mathcal{O}_{\mathbb{P}^{1}}(-2+k) .
$$

Hence $\operatorname{dim} H_{\xi}^{\overline{\mathcal{O}}}=k-1$. Also note that

$$
\operatorname{dim} H_{\bar{\xi}}^{\overline{\mathcal{O}}}=(n-k)-1
$$

by Serre duality, which agrees with the isomorphism in Theorem 4.1 for the unitary case. See also [37] for this kind of computation.

Following these ideas, a more general result holds. 
Proposition 4.4 Let $X$ be a quasi-projective Riemann surface, such that $\bar{X}$ is a curve of genus $g$, and $\mathcal{D}:=\bar{X} \backslash X$ is a reduced effective divisor of cardinality $n$. Let $G:=\pi_{1}(X)$ and fix $\xi \in \mathbb{T}_{G} \backslash\{\mathbf{1}\}$. Let $k$ be the integer that is the negative of the sum of residues associated with $\xi$. Then, $H_{\xi}^{\mathcal{O}} \neq 0$ except in the following cases:

(1) $g=0$ and $n \leq 2$

(2) $g=1$ and $n=0$

(3) $g=0, n \geq 3$ and $k=n-1$ (in particular, $X=X^{\xi}$ )

(4) $g=1, n=1$ and $\xi=\exp (\omega)$ for $\omega \in H^{0}\left(\bar{X}, \Omega_{\bar{X}}^{1}(\log \mathcal{D})\right)=H^{0}\left(\bar{X}, \Omega_{\bar{X}}^{1}\right)=$ $H^{0}\left(\bar{X}, \mathcal{O}_{\bar{X}}\right)$

Proof The first two cases are immediate since $G$ is Abelian.

We recall (see (3-2)) that $H_{\xi}^{\overline{\mathcal{O}}}$ is the cokernel of

$$
A:=H^{0}\left(\bar{X}, \widetilde{L}_{\xi}\right) \stackrel{\bar{\nabla}}{\longrightarrow} H^{0}\left(\bar{X}, \Omega_{\bar{X}}^{1}(\log \mathcal{D}) \otimes \widetilde{L}_{\xi}\right)=: B,
$$

where $\widetilde{L}_{\xi}$ is, as usual, the Deligne extension of $L_{\xi}$ to $\bar{X}$. Recall that $a:=\operatorname{deg} \widetilde{L}_{\xi}=-k$ and $b:=\operatorname{deg} \Omega_{\bar{X}}(\log \mathcal{D}) \otimes \widetilde{L}_{\xi}=2 g-2+n-k$; moreover, $0 \leq k<n$ if $n>0$ and $k=0$ if $n=0$. By Proposition 4.3, $A \neq 0$ if and only if $\widetilde{L}_{\xi} \cong \mathcal{O}_{\bar{X}}$, in particular, $\operatorname{dim} A=1$. We distinguish several cases.

If $g>1$, since $b>0$ then $B \neq 0$. Then, we get the statement when $A=0$. If $A \neq 0$, it is enough to prove that $\operatorname{dim} B>1$. In this case, $B$ is the space of holomorphic sections of $\Omega_{\bar{X}}^{1}(\log \mathcal{D})$, which admits the space of holomorphic 1-forms of $\bar{X}$ as a subspace. Since such a space has dimension $g>1$ we are done.

For $g=1$, we assume $n>0$. Hence $0 \leq k<n$ and $b>0$; if $A=0$ we are done. If $A \neq 0$, we have in particular that $B$ is the space of holomorphic sections of

$$
\Omega_{\bar{X}}^{1}(\log \mathcal{D})=\mathcal{O}_{\bar{X}}(\mathcal{D}) .
$$

If $n \geq 2$, then $\operatorname{dim} B>1$ and we are done. If $n=1$, then $\operatorname{dim} B=1$ and hence $H_{\xi}^{\mathcal{O}}=0$. Note that $\widetilde{L}_{\xi} \cong \mathcal{O}_{\bar{X}}$ is equivalent to the property $\xi=\exp (\omega)$ for $\omega \in$ $H^{0}\left(\bar{X}, \Omega_{\bar{X}}^{1}(\log \mathcal{D})\right)$.

For $g=0$, we assume $n>2$ in which case $b=n-k-2 \geq-1$ and hence $\operatorname{dim} B=$ $n-k-1$. If $k=0$, we have immediately that $\operatorname{dim} B>1=\operatorname{dim} A$. If $k>0$, then $A=0$ and if $k<n-1$, then $\operatorname{dim} B>0$.

Corollary 4.5 Let us assume that $H^{1}\left(X ; \mathbb{C}_{\xi}\right) \neq 0$ and $H_{\xi}^{\mathcal{O}}=0$. Consider $p \in X$, $Y:=X \backslash\{p\}$ and $\xi_{1}$ the induced character in $Y$. Then, $H_{\xi_{1}}^{\mathcal{O}} \neq 0$ (on $Y$ ). 
Proof If $g=1$, it is trivial. If $g=0$, note that the maximum value of $k$ equals $n-1$ and it can be obtained only when $\xi$ ramifies around all the punctures.

Example 4.6 In Example 1.9, we showed that the twisted cohomology of a Riemann surface $X \subset \bar{X}$ with respect to a character $\xi$ that does not ramify around a point $p \in \bar{X}$ changes if we replace $X$ by $X \cup\{p\}$. This example has the same purpose, but is more subtle and points at the root of the relation between the twisted cohomology of a character $\xi$ on $X$ and $\xi_{0}$ on $X^{\xi}$.

Let $\bar{X}$ be an elliptic curve, $p$ a point on it and $X=\bar{X} \backslash\{p\}$. The spaces of characters over $X$ and $\bar{X}$ coincide. Let us fix $\mathbf{1} \neq \xi \in \mathbb{T}_{\pi_{1}(X)}$ and let $\xi_{0}$ be the corresponding character in $\mathbb{T}_{\pi_{1}(\bar{X})}$. Since $\pi_{1}(\bar{X})$ is Abelian, $H^{1}\left(\bar{X} ; \mathbb{C}_{\xi_{0}}\right)=0$.

Let us decompose $\xi=\psi \exp (\omega)$, where $\psi$ is unitary and

$$
\omega \in H^{0}\left(\bar{X}, \Omega_{\bar{X}}^{1}(\log p)\right)=H^{0}\left(\bar{X}, \Omega_{\bar{X}}^{1}\right) .
$$

The Deligne extension of $\psi$ is a degree 0 line bundle $\widetilde{L}_{\psi}$ over $\bar{X}$, which is trivial if and only if $\psi=\mathbf{1}$. The short exact sequence

$$
0 \rightarrow \Omega_{\bar{X}}^{1} \otimes \widetilde{L}_{\psi} \rightarrow \Omega_{\bar{X}}^{1}(\log p) \otimes \tilde{L}_{\psi} \rightarrow \mathbb{C}_{p} \rightarrow 0
$$

induces (note that $\Omega_{\bar{X}}^{1} \cong \mathcal{O}_{\bar{X}}$ )

$0 \rightarrow H^{0}\left(\bar{X} ; \widetilde{L}_{\psi}\right) \rightarrow H^{0}\left(\bar{X} ; \mathcal{O}(p) \otimes \widetilde{L}_{\psi}\right) \rightarrow \mathbb{C}$

$$
\rightarrow H^{1}\left(\bar{X} ; \widetilde{L}_{\psi}\right) \rightarrow H^{1}\left(\bar{X} ; \mathcal{O}(p) \otimes \widetilde{L}_{\psi}\right) \rightarrow 0 .
$$

Let us assume that $\psi \neq \mathbf{1}$. Then, this sequence implies (using Serre Duality and the Riemann-Roch formula)

$H^{0}\left(\bar{X} ; \widetilde{L}_{\psi}\right)=H^{1}\left(\bar{X} ; \widetilde{L}_{\psi}\right)=H^{1}\left(\bar{X} ; \mathcal{O}(p) \otimes \widetilde{L}_{\psi}\right)=0, \quad H^{0}\left(\bar{X} ; \mathcal{O}(p) \otimes \widetilde{L}_{\psi}\right) \cong \mathbb{C}$

Note also that $H^{0}\left(\bar{X} ; \widetilde{L}_{\psi}\right)=0=H^{1}\left(\bar{X} ; \widetilde{L}_{\psi}\right)$. Applying (3-2) and (3-3) we obtain $H_{\xi}^{\mathcal{O}} \cong \mathbb{C}$ and $H_{\xi}^{\overline{\mathcal{O}}}=0$.

If $\psi=\mathbf{1}$ the sequence implies

$$
H^{0}(\bar{X} ; \mathcal{O}) \cong H^{0}(\bar{X} ; \mathcal{O}(p)) \cong H^{1}(\bar{X} ; \mathcal{O}) \cong \mathbb{C}, \quad H^{1}(\bar{X} ; \mathcal{O}(p))=0 .
$$

Since $H^{0}(\bar{X} ; \mathcal{O}) \cong H^{1}(\bar{X} ; \mathcal{O}) \cong \mathbb{C}$, applying (3-2) and (3-3) we obtain $H_{\xi}^{\mathcal{O}}=0$ and $H_{\xi}^{\overline{\mathcal{O}}} \cong \mathbb{C}$.

In both cases we obtain that $H^{1}\left(X ; \mathbb{C}_{\xi}\right) \cong \mathbb{C}$ but the decomposition into the spaces $H_{\xi}^{\mathcal{O}}$ and $H_{\xi}^{\overline{\mathcal{O}}}$ depends on the analytic type of $\bar{X}$. 
The situation described in the previous example holds in a more general setting. We are in position to state the last ingredient needed for the proof of Proposition 4.2.

Proposition 4.7 Let $X$ be a Riemann surface and let $\xi$ be a character on $X$ such that the Deligne extension $\widetilde{L}_{\psi}$ associated with the unitary part of a unitary-holomorphic decomposition of $\xi$ is non-trivial. Then $H_{\xi}^{\overline{\mathcal{O}}}=H_{\xi_{0}}^{\overline{\mathcal{O}}}=H^{1}\left(\bar{X} ; \tilde{L}_{\psi}\right)$ where $\xi_{0}$ is the character induced by $\xi$ on $X^{\xi}$.

Proof Let us consider the chain $X \subset X^{\xi} \subset \bar{X}$ and the reduced divisors $\mathcal{D}:=\bar{X} \backslash X$ and $\mathcal{D}^{\xi}:=\bar{X} \backslash X^{\xi}$. Since the statement is trivial when $\mathcal{D}=\mathcal{D}^{\xi}$ we may assume $\mathcal{D}^{\xi} \subsetneq \mathcal{D}$.

We must consider the sequence (3-3) applied to $\mathcal{D}$ and $\mathcal{D}^{\xi}$ for the Deligne extension $\widetilde{L}_{\psi}$ associated with $\xi=\psi \exp (\omega)$, where $\psi$ is unitary and $\omega \in H^{0}\left(\bar{X} ; \Omega_{\bar{X}}^{1}\left(\log \mathcal{D}^{\xi}\right)\right)$. By hypothesis $\widetilde{L}_{\psi} \not \mathcal{O}_{\bar{X}}$.

This sequence reduces to a morphism, defined by the exterior product by $\omega$, where the source $H^{1}\left(\bar{X} ; \widetilde{L}_{\psi}\right)$ is common for $\mathcal{D}$ and $\mathcal{D}^{\xi}$. Let us study the targets. For $\mathcal{D}$ we have

$$
H^{1}\left(\bar{X} ; \Omega_{\bar{X}}^{1}(\log \mathcal{D}) \otimes \widetilde{L}_{\psi}\right),
$$

which is isomorphic, by Serre Duality, to the dual of $H^{0}\left(\bar{X} ; \mathcal{O}(-\mathcal{D}) \otimes \widetilde{L}_{\psi}^{-1}\right)$. Since $\operatorname{deg} \mathcal{D}>0$ and $\operatorname{deg} \widetilde{L}_{\psi}^{-1}<\operatorname{deg} \mathcal{D}$ (see Proposition 4.3), this space is trivial.

Let us now consider $H^{1}\left(\bar{X} ; \Omega_{\bar{X}}^{1}\left(\log \mathcal{D}^{\xi}\right) \otimes \widetilde{L}_{\psi}\right)$, ie, by duality

$$
H^{0}\left(\bar{X} ; \mathcal{O}\left(-\mathcal{D}^{\xi}\right) \otimes \tilde{L}_{\psi}^{-1}\right) .
$$

Note that the degree $e$ of this line bundle is non-positive. If $e<0$, then the space is trivial again. If $e=0$, then we have $\mathcal{D}^{\xi}=\varnothing$; since $\tilde{L}_{\psi}$ is non-trivial, the space is also trivial.

Then, $H_{\xi}^{\overline{\mathcal{O}}}=H^{1}\left(\bar{X} ; \tilde{L}_{\psi}\right)=H_{\xi_{0}}^{\overline{\mathcal{O}}}$.

Proof of Proposition 4.2 The result is trivial for unitary characters, so we assume that $\xi$ is non-unitary. We break the proof in several steps.

Step 1 The case when $X$ is a Riemann surface

After Proposition 4.7 it is enough to prove that either $\xi$ or $\bar{\xi}$ admits a unitaryholomorphic decomposition such that the Deligne extension $\widetilde{L}_{\psi}$ associated with the unitary part is non-trivial. 
Let us assume that this is not the case for $\xi$. Then, $\mathcal{D}^{\xi}=\varnothing, \bar{X}=X^{\xi}$ (see Remark 3.10), the unitary part of $\xi$ is 1 (see Proposition 4.3) and $\xi=\exp (\omega)$, where $0 \neq \omega \in$ $H^{0}\left(\bar{X} ; \Omega_{\bar{X}}^{1}\right)$. We deduce that $\bar{\xi}=\exp (\bar{\omega})$, where $\bar{\omega} \in H^{1}\left(\bar{X} ; \mathcal{O}_{\bar{X}}\right)$.

Let us consider the decomposition $\bar{\omega}=\alpha+\beta$ associated with

$$
H^{1}(\bar{X} ; \mathbb{C})=2 \sqrt{-1} \pi H^{1}(\bar{X} ; \mathbb{R}) \oplus H^{0}\left(\bar{X} ; \Omega_{\bar{X}}^{1}\right) .
$$

Note that the spaces

$$
H_{\xi}^{\overline{\mathcal{O}}} \quad \text { and } \quad H_{\xi_{0}}^{\overline{\mathcal{O}}}
$$

do not change if we replace $\xi$ by $\exp (t \omega), t \in \mathbb{R}^{*}$; we may assume that $\alpha \notin$ $2 \sqrt{-1} \pi H^{1}(\bar{X} ; \mathbb{Z})$ and the unitary-holomorphic decomposition $\bar{\xi}=\exp (\alpha) \exp (\beta)$ satisfies the hypothesis in Proposition 4.7. The result follows for $\bar{\xi}$ and we have achieved Step 1.

Step 2 Preparation of an induction process when $X$ is a quasi-projective surface

Using the arguments of Step 1, we obtain that either $\xi$ or $\bar{\xi}$ fits in a unitary-holomorphic decomposition such that the Deligne extension of the unitary part is non-trivial. We can assume that this is the case for $\xi=\psi \exp (\omega), \psi$ unitary with $\widetilde{L}_{\psi} \not \mathcal{O}_{\bar{X}}$ (in particular, $\psi \neq \mathbf{1})$ and $\omega \in H^{0}\left(\bar{X} ; \Omega_{\bar{X}}^{1}\left(\log \mathcal{D}^{\xi}\right)\right)$.

We prove the statement by induction on the number $n$ of irreducible components of $\mathcal{D}^{\prime}:=\mathcal{D}-\mathcal{D}^{\xi}$. If $n=0$ the statement is trivially true. Let us assume $n>0$ and let us fix an irreducible component $D$ of $\mathcal{D}^{\prime}$. Let us denote $\mathcal{D}^{\prime \prime}:=\mathcal{D}-D$ and $\xi^{\prime \prime}$ the character induced by $\xi$ on $X^{\prime \prime}:=\bar{X} \backslash \mathcal{D}^{\prime \prime}$. Let $\check{D}:=D \backslash \mathcal{D}^{\prime \prime}$. Also set

$$
\mathcal{L}^{\prime \prime}=\Omega_{\bar{X}}^{1}\left(\log \mathcal{D}^{\prime \prime}\right) \otimes \widetilde{L}_{\psi}, \quad \mathcal{L}=\Omega_{\bar{X}}^{1}(\log \mathcal{D}) \otimes \widetilde{L}_{\psi} .
$$

Since $H^{0}\left(\bar{X}, \widetilde{L}_{\psi}\right)$, we have:

$$
\begin{aligned}
& H_{\xi}^{\mathcal{O}}=\operatorname{ker}\left(H^{0}(\bar{X}, \mathcal{L}) \stackrel{\wedge \omega}{\longrightarrow} H^{0}\left(\bar{X}, \Omega_{\bar{X}}^{2}(\log \mathcal{D}) \otimes \widetilde{L}_{\psi}\right)\right) \\
& H_{\xi^{\prime \prime}}^{\mathcal{O}}=\operatorname{ker}\left(H^{0}\left(\bar{X}, \mathcal{L}^{\prime \prime}\right) \stackrel{\wedge \omega}{\longrightarrow} H^{0}\left(\bar{X}, \Omega_{\bar{X}}^{2}\left(\log \mathcal{D}^{\prime \prime}\right) \otimes \widetilde{L}_{\psi}\right)\right) \\
& H_{\xi}^{\overline{\mathcal{O}}}=\operatorname{ker}\left(H^{1}\left(\bar{X}, \widetilde{L}_{\psi}\right) \stackrel{\wedge \omega}{\longrightarrow} H^{1}(\bar{X}, \mathcal{L})\right) \\
& H_{\xi^{\prime \prime}}^{\overline{\mathcal{O}}}=\operatorname{ker}\left(H^{1}\left(\bar{X}, \widetilde{L}_{\psi}\right) \stackrel{\wedge \omega}{\longrightarrow} H^{1}\left(\bar{X}, \mathcal{L}^{\prime \prime}\right)\right)
\end{aligned}
$$

Both $\mathcal{L}$ and $\mathcal{L}^{\prime \prime}$ fit in the following short exact sequence

$$
0 \rightarrow \mathcal{L}^{\prime \prime} \rightarrow \mathcal{L} \rightarrow i_{*}\left(\tilde{L}_{\psi}\right)_{\mid D} \rightarrow 0
$$


and the associated long exact sequence

$0 \longrightarrow H^{0}\left(\bar{X}, \mathcal{L}^{\prime \prime}\right) \longrightarrow H^{0}(\bar{X}, \mathcal{L}) \stackrel{(\text { () })}{\longrightarrow} H^{0}\left(D ;\left(\tilde{L}_{\psi}\right) \mid D\right) \stackrel{(*)}{\longrightarrow} H^{1}\left(\bar{X}, \mathcal{L}^{\prime \prime}\right) \longrightarrow H^{1}(\bar{X}, \mathcal{L})$.

Step 3 If the map $(*)$ of (4-1) vanishes then $H_{\xi}^{\overline{\mathcal{O}}}=H_{\xi^{\prime \prime}}^{\overline{\mathcal{O}}}$.

By the exactness of (4-1) the map $H^{1}\left(\bar{X}, \mathcal{L}^{\prime \prime}\right) \rightarrow H^{1}(\bar{X}, \mathcal{L})$ is injective; since $\wedge \omega$ factorizes through this mapping in the definition of $H_{\xi}^{\overline{\mathcal{O}}}$, it is immediate that $H_{\xi}^{\overline{\mathcal{O}}}=H_{\xi^{\prime \prime}}^{\overline{\mathcal{O}}}$. We have proved Step 3.

Step 4 If the restriction of $\psi$ to $\check{D}$ is non-trivial then $H_{\xi}^{\overline{\mathcal{O}}}=H_{\xi^{\prime \prime}}^{\overline{\mathcal{O}}}$.

The third term $H^{0}\left(D ;\left(\tilde{L}_{\psi}\right)_{\mid D}\right)$ of (4-1) vanishes by Proposition 4.3 and hence, also $(*)$ does. The statement of Step 3 implies Step 4.

Assumption According to Step 4, from now on we assume that the restriction of $\psi$ to $\check{D}$ is trivial.

The character $\psi$ acts trivially on $\pi_{1}(\check{D})$. By Proposition $4.3\left(\tilde{L}_{\psi}\right)_{\mid D} \cong \mathcal{O}_{D}$. Since $H^{0}\left(D ; \mathcal{O}_{D}\right) \cong \mathbb{C}$, either $(\boldsymbol{A})$ or $(*)$ vanishes, ie, either

$$
H_{\xi}^{\mathcal{O}} \cong H_{\xi^{\prime \prime}}^{\mathcal{O}} \quad \text { or } \quad H_{\xi}^{\overline{\mathcal{O}}}=H_{\xi^{\prime \prime}}^{\overline{\mathcal{O}}}
$$

Step 5 If $\operatorname{dim} H^{1}\left(X ; \mathbb{C}_{\psi}\right)=\operatorname{dim} H^{1}\left(X^{\prime \prime} ; \mathbb{C}_{\psi^{\prime \prime}}\right)$ then $H_{\xi}^{\overline{\mathcal{O}}}=H_{\xi^{\prime \prime}}^{\overline{\mathcal{O}}}$.

This is immediate from the above property.

Assumption According to Step 5, from now on we will assume $\operatorname{dim} H^{1}\left(X ; \mathbb{C}_{\xi}\right)>$ $\operatorname{dim} H^{1}\left(X^{\prime \prime} ; \mathbb{C}_{\xi^{\prime \prime}}\right)$.

Step $6 \operatorname{dim} H^{1}\left(X ; \mathbb{C}_{\psi}\right)-\operatorname{dim} H^{1}\left(X^{\prime \prime} ; \mathbb{C}_{\psi^{\prime \prime}}\right)=1$

The above arguments imply $\operatorname{dim} H^{1}\left(X ; \mathbb{C}_{\xi}\right)-\operatorname{dim} H^{1}\left(X^{\prime \prime} ; \mathbb{C}_{\xi^{\prime \prime}}\right)=1$. By duality

$$
\operatorname{dim} H_{1}\left(X ; \mathbb{C}_{\xi^{-1}}\right)-\operatorname{dim} H_{1}\left(X^{\prime \prime} ; \mathbb{C}_{\left(\xi^{\prime \prime}\right)^{-1}}\right)=1 .
$$

Using a Mayer-Vietoris exact sequence for $H_{1}\left(X^{\prime \prime} ; \mathbb{C}_{\xi}\right)$, we obtain that a meridian $\gamma$ around $D$ defines a twisted cycle $\zeta_{D}$ which determines a non-trivial homology class in the kernel of the natural map $H_{1}\left(X ; \mathbb{C}_{\xi}\right) \rightarrow H_{1}\left(X^{\prime \prime} ; \mathbb{C}_{\xi^{\prime \prime}}\right)$ induced by inclusion. 
More precisely, let $N$ be a regular neighborhood of $\check{D}$, and note that $X^{\prime \prime}=X \cup N$. Then $X \cap N$ has the homotopy type of a Seifert 3-manifold $M$, in fact a circle bundle over $\check{D}$ and $N$ has the homotopy type of $\check{D}$. The character is trivial on both $M$ and $\check{D}$; recall that $\operatorname{dim} H_{1}(M, \mathbb{C}) \geq \operatorname{dim} H_{1}(\check{D} ; \mathbb{C})$ and equality arises only when $\check{D}$ is compact and its Euler number is non-zero. Since the map $H_{1}\left(X, \mathbb{C}_{\xi}\right) \rightarrow H_{1}\left(X^{\prime \prime}, \mathbb{C}_{\xi^{\prime \prime}}\right)$ is surjective, the exact sequence

$$
H_{1}\left(M, \mathbb{C}_{\xi}\right) \rightarrow H_{1}\left(X, \mathbb{C}_{\xi}\right) \oplus H_{1}\left(\check{D}, \mathbb{C}_{\xi}\right) \rightarrow H_{1}\left(X^{\prime \prime}, \mathbb{C}_{\xi^{\prime \prime}}\right) \rightarrow 0
$$

can be completed to a short exact sequence and $\operatorname{dim} H_{1}(M, \mathbb{C})=\operatorname{dim} H_{1}(\check{D} ; \mathbb{C})+1$. These arguments also hold for the characters $\xi_{t}:=\psi \exp (t \omega), t \in \mathbb{C}^{*}$. Since the character $\psi$ is limit of the characters $\xi_{t}$, we obtain that the class of $\zeta_{D}$ is a non-trivial element of $H_{1}\left(X ; \mathbb{C}_{\psi}\right)$; since the difference of dimensions is at most one, Step 6 is achieved.

Step 7 The map ( $)$ is non-trivial.

As stated in Remark 3.4, for the character $\psi$ the decomposition is natural and we can evaluate the elements of

$$
H_{\psi}^{\overline{\mathcal{O}}} \text { in } H_{1}\left(X ; \mathbb{C}_{\psi}\right) \text { and } H_{\psi^{\prime \prime}}^{\overline{\mathcal{O}}} \text { in } H_{1}\left(X^{\prime \prime} ; \mathbb{C}_{\psi^{\prime \prime}}\right)
$$

Both spaces are equal to $H^{1}\left(\bar{X}, \widetilde{L}_{\psi}\right)$. Since $\zeta_{D}$ is a trivial cycle of $H_{1}\left(X^{\prime \prime} ; \mathbb{C}_{\psi^{\prime \prime}}\right)$, $\forall \alpha \in H^{1}\left(\bar{X}, \tilde{L}_{\psi}\right)$, we have that $\alpha\left(\zeta_{D}\right)=0$. Using the duality between twisted homology and cohomology, there exists

$$
\beta \in H_{\psi}^{\mathcal{O}}=H^{0}\left(D ;\left(\tilde{L}_{\psi}\right)_{\mid D}\right)
$$

such that $\beta(\gamma) \neq 0$. Then $\beta$ is an element of $H^{0}(\bar{X}, \mathcal{L}) \backslash H^{0}\left(\bar{X}, \mathcal{L}^{\prime \prime}\right)$. The exactness of (4-1) implies the statement of Step 7.

We conclude that $(*)$ vanishes and hence $H_{\xi}^{\overline{\mathcal{O}}} \cong H_{\xi^{\prime \prime}}^{\overline{\mathcal{O}}}$. One can replace $X$ by $X^{\prime \prime}$ and apply the induction hypothesis once again.

\section{Characters and orbifold maps}

The main tool for the proof of Theorem 1 is the following result, which corresponds to [1, Proposition V.1.4], except for this statement a weaker hypothesis is required (only non-torsion characters as opposed to non-unitary). In this section the notation used in Sections 1 and 2 will be followed, that is, $X$ is a smooth quasi-projective surface, $G:=\pi_{1}(X), \bar{X}$ is a smooth projective compactification of $X$ such that $\mathcal{D}:=\bar{X} \backslash X$ is a normal crossing divisor and $C_{\varphi}$ is an orbifold coming from a Riemann surface $C$. 
Theorem 5.1 Let $\xi \in \mathbb{T}_{G}$ be a non-torsion character in $\Sigma_{1}(X) \neq \varnothing$ and $0 \neq \theta \in$ $H^{1}\left(X ; \mathbb{C}_{\xi}\right)$. Then there exists

- $\quad$ an orbifold $C_{\varphi}$,

- an orbifold map $f: X \rightarrow C_{\varphi}$,

- a character $\xi_{C} \in \Sigma_{1}\left(C_{\varphi}\right)$,

- a cohomology class $\theta_{C} \in H^{1}\left(C_{\varphi} ; \mathbb{C}_{\xi_{C}}\right)$,

such that $\xi=f^{*}\left(\xi_{C}\right)$ and $\theta=f^{*}\left(\theta_{C}\right)$.

Lemma 5.2 It is enough to prove Theorem 5.1 for holomorphically pure elements in $H^{1}\left(X ; \mathbb{C}_{\xi}\right)$.

Proof First note that if Theorem 5.1 is true for holomorphically pure elements in $H^{1}\left(X ; \mathbb{C}_{\xi}\right)$, then it is also true for anti-holomorphically pure elements by Theorem 4.1 and Proposition 4.2.

Let us assume that the claim in Theorem 5.1 holds for holomorphically pure elements but not in general. Then there exist non-zero $\theta_{1}, \alpha_{2} \in H^{1}\left(X ; \mathbb{C}_{\xi}\right)$, where $\theta_{1}$ is holomorphically pure, and $\alpha_{2}$ is anti-holomorphically pure, coming from different orbifold morphisms, say $f_{1}: X \rightarrow C_{1}$ and $f_{2}: X \rightarrow C_{2}$ respectively. We may replace $C_{2}$ by $C_{2} \backslash\{p\}\left(p \in C_{2}\right)$ and $X$ by $f_{2}^{-1}\left(C_{2} \backslash\{p\}\right)$. By Corollary 4.5 and Proposition 2.13 there exists a holomorphically pure element $\theta_{2} \neq 0$ that is a pull-back by $f_{2}$.

Since $\theta_{1}$ is a twisted logarithmic $1-$ form, $p \mapsto \operatorname{ker}\left(\theta_{1}\right)_{p}=\operatorname{ker}\left(d f_{1}\right)_{p}$ defines a foliation $\mathcal{F}_{1}$, which determines and is determined by $f_{1}$. Analogously, we construct a foliation $\mathcal{F}_{2} \neq \mathcal{F}_{1}$. Choose a point $q \in X$ such that $\left(\mathcal{F}_{2}\right)_{q} \neq\left(\mathcal{F}_{1}\right)_{q}$. Let $\left(t_{1}, t_{2}\right) \in \mathbb{C}^{2} \backslash\{(0,0)\}$ and consider the holomorphically pure element $\theta_{\left(t_{1}, t_{2}\right)}:=t_{1} \theta_{1}+t_{2} \theta_{2}$. Since we claim that Theorem 5.1 holds for holomorphically pure elements and two proportional elements come from the same orbifold morphism, for any $\left[t_{1}: t_{2}\right] \in \mathbb{P}^{1}$ we obtain an orbifold morphism $f_{\left[t_{1}: t_{2}\right]}: X \rightarrow C_{\left[t_{1}: t_{2}\right]}$ such that for generic $p \in X$ we have $\operatorname{ker}\left(\theta_{\left(t_{1}, t_{2}\right)}\right)_{p}=\operatorname{ker}\left(d f_{\left[t_{1}: t_{2}\right]}\right)_{p}$. Since these morphisms have distinct fibers we have obtained a family of pairwise non-equivalent orbifold morphisms onto a hyperbolic orbifold parametrized by $\mathbb{P}^{1}$. The set of equivalence classes of such morphisms is at most countable (see [1, Lemma V.1.5]) and we obtain a contradiction.

The following key result is the orbifold version of [1, Proposition V.1.4], which in turn is the quasi-projective version of [8, Proposition 2.1]. We adapt Arapura's proofs [1, Propositions V.1.3, V.1.4] stressing the details required for the orbifold version. 
Proposition 5.3 Let $\xi=\psi \exp (\omega) \in \mathbb{T}_{G}$ be a strict unitary-holomorphic decomposition of the character $\xi$ and let $\theta \in H^{1}\left(X ; \mathbb{C}_{\xi}\right)$ be a holomorphically pure element. Then, there exists

(1) an orbifold map $f: X \rightarrow C_{\varphi}$,

(2) $\xi_{C} \in \mathbb{T}_{\Pi}, \Pi:=\pi_{1}^{\text {orb }}\left(C_{\varphi}\right)$,

(3) $\theta_{C} \in H^{1}\left(C_{\varphi} ; \mathbb{C}_{\xi_{C}}\right)$,

such that $\xi=f^{*}\left(\xi_{C}\right)$ and $\theta=f^{*}\left(\theta_{C}\right)$.

Proof Recall that the Deligne extension $\widetilde{L}_{\psi}$ is an extension for $L_{\xi}$ with a meromorphic connection $\nabla_{\omega}$. Using (3-2) the pull-back $\theta$ can be represented by a section $\eta \in H^{0}\left(\bar{X} ; \Omega_{\bar{X}}^{1}(\log \mathcal{D}) \otimes \widetilde{L}_{\psi}\right)$ such that $\nabla_{\omega}(\eta)=0$ and it is not the image by $\nabla_{\omega}$ of a holomorphic section of $\tilde{L}_{\psi}$.

According to Theorem 3.3, $\eta$ is so that $\eta \wedge \omega=0$. In addition, if $\widetilde{L}_{\psi}=\mathcal{O}_{\bar{X}}$, then $\eta$ is not a complex multiple of $\omega$.

Using [1, Proposition V.1.3], a holomorphic mapping $f: X \rightarrow C$ onto a quasi-projective Riemann surface $C$ exists with the following properties:

(1) The mapping is the restriction of $\bar{f}: \bar{X} \rightarrow \bar{C}$ (possibly after performing additional blow-ups on $\bar{X}$ ), $\mathcal{D}_{1}:=\bar{C} \backslash C$.

(2) There is a logarithmic 1 -form $\omega_{1} \in H^{0}\left(\bar{C} ; \Omega_{\bar{C}}^{1}\left(\log \mathcal{D}_{1}\right)\right)$ such that $\omega=\bar{f}^{*}\left(\omega_{C}\right)$.

At this point it is worth mentioning that [1, Proposition V.1.3] also ensures the existence of a character on $C$, whose pull-back by $f$ translated by a torsion element, equals $\psi$. Alternatively, we will use a more detailed description of $\psi$ to describe it as the pull-back of a character on a certain orbifold structure on $C$.

Let $\varphi$ be the maximal orbifold structure on $C$ naturally induced by $f$ as described in Remark 2.5, and let $\check{C}$ be the set of non-multiple points for $\varphi$. Let us write $\bar{C} \backslash \check{C}=\mathcal{D}_{1}+\mathcal{D}_{2}, \check{X}:=f^{-1}(\check{C})$ and $\check{f}:=f_{\mid}: \check{X} \rightarrow \check{C}$. Let $\check{\psi}$ denote the induced character by $\psi$ on $\check{X}$.

Let us consider $F$ a generic fiber of $\check{f}$ and consider its closure $\bar{F}$ in $\bar{X}$. Outside the multiple fibers there is an exact sequence (see [38, Lemma 1.5C; 17])

$$
\pi_{1}(F) \rightarrow \pi_{1}(\check{X}) \rightarrow \pi_{1}(\check{C}) \rightarrow 1 .
$$

In order to check that $\check{\psi}_{\check{ }}$ is the pullback of a character $\psi_{\check{C}}$ of $\pi_{1}(\check{C})$ it is enough to check that $\pi_{1}(F) \subset \operatorname{ker} \check{\psi}$. Arapura proceeds as follows: there is a meromorphic section $\beta$ of $\widetilde{L}_{\psi}$ such that $\eta=\omega \otimes \beta$. Since $F$ is generic, one may assume $\omega_{\mid \bar{F}} \neq 0$. Hence, $\beta_{\mid \bar{F}}$ is holomorphic. This fact has two consequences according to Proposition 4.3 applied to $F$ : 
- $\left(\tilde{L}_{\psi}\right)_{\mid \bar{F}}=\mathcal{O}_{\bar{F}}$ and hence $\psi$ and $\check{\psi}$ are trivial on $F$, thus $\check{\psi}$ is a pullback, say $\check{\psi}=\check{f}^{*}\left(\psi_{\check{C}}\right)$. It follows that $\check{\xi}:=\check{\psi} \exp (\omega)$ is also a pullback, say $\check{\xi}=\check{f}^{*}\left(\xi_{\check{C}}\right)$, where $\xi_{\check{C}}=\psi_{\check{C}} \exp \left(\omega_{\check{C}}\right)$.

- The meromorphic section $\beta$ is holomorphic and constant on $\bar{F}$, ie, $\beta$ is a pullback by $\bar{f}$, say $\beta=\bar{f}^{*}\left(\beta_{\bar{C}}\right)$. Hence $\eta$ is the pullback by $\bar{f}$ of a logarithmic form, that is $\eta=\bar{f}^{*}\left(\omega_{\bar{C}} \otimes \beta_{\bar{C}}\right)$ (with poles along $\left.\mathcal{D}_{1}+\mathcal{D}_{2}\right)$.

- Moreover, the character $\psi_{\check{C}}$ induces a character $\psi_{C_{\varphi}}$ on $C_{\varphi}$. In order to see this, note that, if $f^{*}(p)=\sum n_{i} E_{i}, \mu_{i}$ is a meridian around $E_{i}$ and $\mu_{p}$ is a meridian around $p$, then

$$
1=\psi\left(\mu_{i}\right)=\check{\psi}\left(\mu_{i}\right)=\psi_{\check{C}}\left(\mu_{p}^{n_{i}}\right)=\left(\psi_{\check{C}}\left(\mu_{p}\right)\right)^{n_{i}} .
$$

Therefore $\left(\psi_{\check{C}}\left(\mu_{p}\right)\right)^{\varphi(p)}=1$, since by construction $\varphi(p)=\operatorname{gcd}\left(n_{i}\right)$.

The existence of $\check{C}, \xi_{\check{C}}$ and $\theta_{\check{C}}$ proves the result for $\check{X}$ with respect to a Riemann surface. Finally, the discussion above and Proposition 2.13 show the existence of $C_{\varphi}$, $\xi_{C}$ and $\theta_{C}$, which proves the result for $X$ with respect to orbifolds.

The non-unitary case for Theorem 5.1 is partially proved in [1, Proposition V.1.4]. In fact, the argumentation line in the proof only establishes the statement for characters that ramify over all components of the divisor $\mathcal{D}=\bar{X} \backslash X$. More precisely, since Theorem 4.1 is needed, Arapura replaces the quasi-projective manifold $X$ by the bigger manifold $X^{\xi} \subset \bar{X}$ but, as Examples 1.9 and 4.6 show, this must be done carefully. Proposition 4.2 solves these issues.

Corollary 5.4 Theorem 5.1 is true when the character $\xi$ is either non-unitary or $b_{1}\left(X^{\xi}\right)>b_{1}(\bar{X})$.

Proof It is a direct consequence of Proposition 5.3 and Lemmas 5.2 and 3.12.

Remark 5.5 This corollary is enough if we restrict our attention to the case when $b_{1}(\bar{X})=0$ as in [37].

The remaining case, that is, non-torsion unitary characters $\xi$ such that $b_{1}\left(X^{\xi}\right)=b_{1}(\bar{X})$, will be treated with different arguments, following Delzant's technique in [24] for Kähler manifolds. This strategy uses results by Levitt [35] and Simpson [41], which can be adapted to our case.

Proposition 5.6 Let $\xi$ be a non-torsion unitary character such that $b_{1}\left(X^{\xi}\right)=b_{1}(\bar{X})$ and let $0 \neq \theta \in H^{1}\left(X ; \mathbb{C}_{\xi}\right)$. Then, Theorem 5.1 holds. 
Proof Let us assume that $\xi$ is not algebraic. Since $\Sigma_{k}:=\Sigma_{k}(X)$ is an algebraic subvariety of $\mathbb{T}_{G}, G=\pi_{1}(X)$, defined with rational coefficients, one can deduce that $\xi$ cannot be an isolated point of $\Sigma_{k}$. Hence, if $k=\operatorname{dim} H^{1}\left(X ; \mathbb{C}_{\xi}\right)$ and $V$ is the irreducible component of $\Sigma_{k}$ containing $\xi$, this component contains non-unitary elements and the result follows easily.

From now on, $\xi$ will be assumed to be algebraic. If $\xi(G)$ only contains algebraic integers, two cases may happen: either all the conjugates of these algebraic integers are also algebraic integers, or not. In the first case the Kronecker Theorem implies that $\xi$ is torsion and this case has been excluded. In the second case, a conjugate character $\widetilde{\xi}$ is non-integral. Since all the statements are algebraic, it is enough to prove the result for $\tilde{\xi}$.

Then, we can assume $\xi$ is algebraic and non-integral and we can follow the proof of [24, Proposition 2]. The first step is to construct an exceptional class $\omega \in H^{1}(X ; \mathbb{R}) \backslash\{0\}$ in the sense of Bieri, Neumann and Strebel [9]; in fact, $\omega$ is defined with integer coefficients. We identify $H^{1}(X ; \mathbb{R})$ with $\operatorname{Hom}(G, \mathbb{R})$.

We sketch the construction of $\omega$; see [24] for more details. Since $H^{1}\left(X ; \mathbb{C}_{\xi}\right)$ is generated by elements in a number field we may assume that $\theta$ is represented by a cocycle (see Section 1.4) in a number field (also denoted by $\theta$ ). We fix such a number field $\mathbb{K}$ in order to have that $\forall g \in G$ the matrix $\left(\begin{array}{c}\xi(g) \\ 0\end{array} \begin{array}{l}\theta(g) \\ 1\end{array}\right)$ has coefficients in $\mathbb{K}$. This defines an action of $G$ on $\mathbb{K}^{2}$ and $\mathbb{P}^{1}(\mathbb{K})$. The fact that $\xi$ is not integral guarantees the existence of a valuation $v$ such that $\omega:=v \circ \xi$ is not trivial. This element is the Busemann cocycle of an exceptional action of $G$ on the Bruhat-Tits tree $T_{\nu}$ (see [10]). As a consequence, the class $\omega$ is exceptional.

Since $\omega:=v \circ \xi$, we deduce that $\operatorname{ker} \xi \subset \operatorname{ker} \omega$. As a consequence, $\omega$ is the restriction of $0 \neq \omega_{\xi} \in H^{1}\left(X^{\xi} ; \mathbb{R}\right)$. We can strengthen this argument since the target of $v$ is an ordered group, without torsion. Thus for any $g \in G$ whose image $\xi(g)$ is a torsion element, one has that $g \in \operatorname{ker} \omega$. Hence, let $\mu$ be the meridian of an irreducible component of $\mathcal{D}^{\xi}$. Since $b_{1}\left(X^{\xi}\right)=b_{1}(\bar{X}), \xi(\mu)$ is a torsion element and, applying the last comment, $\mu \in \operatorname{ker} \omega$. Hence, there exists $\omega_{\bar{X}} \in H^{1}(\bar{X} ; \mathbb{R}) \backslash\{0\}$ such that $\omega_{\xi}$ is the restriction of $\omega_{\bar{X}}$ (in the same way, $\omega$ is the restriction of $\omega_{\bar{X}}$ ). We represent $\omega_{\bar{X}}$ by a closed differential $1-$ form (and $\omega$ by its restriction to $X$ ). For the sake of simplicity we keep the notation of $\omega_{\bar{X}}$ and $\omega$ for the differential 1-forms.

Let us consider an unramified Abelian covering $\bar{\pi}: \bar{X}_{\omega} \rightarrow \bar{X}$ such that $\bar{\pi}^{*} \omega_{\bar{X}}$ is an exact 1 -form (eg, the one determined by $\operatorname{ker} \omega_{\bar{X}}$ as a character of $\left.\pi_{1}(\bar{X})\right)$; let $\bar{F}: X_{\omega} \rightarrow \mathbb{R}$ a primitive of $\bar{\pi}^{*} \omega_{\bar{X}}$. Let $X_{\omega}:=\bar{\pi}^{-1}(X)$; since $\pi_{1}(X) \rightarrow \pi_{1}(\bar{X})$ is surjective the manifold $X_{\omega}$ is connected. Let us denote by $\pi: X_{\omega} \rightarrow X$ the induced covering and $F: X_{\omega} \rightarrow \mathbb{R}$ the restriction of $\bar{F} ; F$ is a primitive of $\pi^{*} \omega$. 
Following [35], we have a characterization of exceptional elements of $H^{1}(X ; \mathbb{R})$ when they are represented by 1 -forms: $\omega$ is exceptional if and only if $F^{-1}\left(\mathbb{R}_{>0}\right)$ has more than one connected component where $F$ is unbounded. Since $\bar{X} \backslash X$ is a union of real codimension 2 varieties the number of connected components of $F^{-1}\left(\mathbb{R}_{>0}\right)$ and $\bar{F}^{-1}\left(\mathbb{R}_{>0}\right)$ is the same. We conclude that $\omega_{\bar{X}}$ is also exceptional.

Using Simpson's results [40], one obtains a surjective morphism $\bar{f}: \bar{X} \rightarrow \bar{C}$ with connected fibers, where $\bar{C}$ is a compact Riemann surface. Let $C:=\bar{f}(\bar{C})$ and let $f:=\overline{f_{1}}: X \rightarrow C$ be the restriction morphism. Here $C$ is considered with the maximal orbifold structure $\varphi$ defined by $f$ (see Remark 2.5). We follow again the arguments of the proof of [24, Proposition 2]. For this orbifold $C_{\varphi}$, the character $\xi$ is in the pull-back of the characteristic variety $\Sigma\left(C_{\varphi}\right)$. This is proved using the action on the tree and showing that the action on the projective line $\mathbb{P}^{1}(\mathbb{K})$ is trivial. As a consequence $\theta$ is in the pull-back of $\Sigma\left(C_{\varphi}\right)$.

Proof of Theorem 5.1 Let $\xi \in \mathbb{T}_{G}$ be a character on $X$. If $\xi$ is not unitary, then Corollary 5.4 gives the result. Hence we might assume that $\xi$ is a unitary character.

On the other hand, if $b_{1}\left(X^{\xi}\right)>b_{1}(\bar{X})$, then again Corollary 5.4 gives the result. Finally, if $b_{1}\left(X^{\xi}\right)=b_{1}(\bar{X})$, then Proposition 5.6 shows the statement.

Remark 5.7 Note that the proof of Theorem 5.1 in fact shows a sharper result, namely, it is also true for unitary characters (torsion or non-torsion) such that $b_{1}\left(X^{\xi}\right)>b_{1}(\bar{X})$. In other words, the only case missing is unitary torsion characters for which $b_{1}\left(X^{\xi}\right)=$ $b_{1}(\bar{X})$. In fact, the statement is not true in general for this case as illustrated in Example 7.3.

Proof of Theorem 1 Once Theorem 5.1 is proved, the arguments of [1, Section V] give the result (they formally apply to $\Sigma_{k}$ for any $k$ ).

\section{Behavior of torsion characters and further applications}

The results of Section 5 do not apply to general torsion characters of a quasi-projective manifold $X$. Some of them may appear as isolated points of some characteristic variety $\Sigma_{k}(X)$ and in that case they may fall either in case (1) or in case (2) of Theorem 1. Since the irreducible components of Theorem 1(1) are torsion-translated subtori of $\mathbb{T}_{G}$, $G:=\pi_{1}(X)$, even if Theorem 5.1 does not apply to torsion characters, the properties of close non-torsion points imply that some of the elements of $H^{1}\left(X ; \mathbb{C}_{\xi}\right)$, for $\xi$ torsion, do come from an orbifold map. In this section we study the behavior of torsion characters as well as show some properties of characteristic varieties that can be derived from Theorem 1. 
Proposition 6.1 Let $\xi \in \mathbb{T}_{G}$ be a torsion character such that $b_{1}\left(X^{\xi}\right)>b_{1}(\bar{X})$. Then $\xi$ cannot be an isolated component of any characteristic variety of $X$.

Proof It is a direct consequence of Lemma 3.12 and Corollary 5.4 that there is a strict unitary-holomorphic decomposition $\xi=\psi \exp (\omega)$. Let us assume that $\xi \in \Sigma_{k}(X)$. Following Remark 3.10 and the ideas in [8] and in [1, Proposition V.1.3], for any $t \in \mathbb{C}$, we have $\xi_{t}:=\xi=\psi \exp (t \omega) \in \Sigma_{k}(X)$, and then $\xi$ cannot be an isolated point of $\Sigma_{k}(X)$.

Next we give a generalization of the results about essential coordinate components of the characteristic varieties of the complement of hypersurfaces in $\mathbb{P}^{n}$; see $[2 ; 36]$. Let $X$ be a quasi-projective manifold and let $V$ be an irreducible component of $\Sigma_{k}(X)$. Let $X^{V}$ be the maximal subvariety $X \subset X^{V} \subset \bar{X}$ such that any character in $V$ is defined over $X^{V}$. Recall that $H^{1}\left(X^{V} ; \mathbb{C}^{*}\right) \subset H^{1}\left(X ; \mathbb{C}^{*}\right)$ and that $V \subset H^{1}\left(X^{V} ; \mathbb{C}^{*}\right)$. The following result is a generalization of a Libgober's result in [36, Lemma 1.4.3].

Proposition 6.2 If $V$ is not contained in $\Sigma_{k}\left(X^{V}\right)$ then $V$ is a torsion point not of pencil type (see Theorem 1).

Proof If $V$ is not isolated then it comes from an orbifold map $X \rightarrow C_{\varphi}$. Following the ideas in [36, Lemma 1.4.3] we can extend this map to $X^{V}$ using the definition of this variety.

We recall a definition introduced in [5;29].

Definition 6.3 For $V$ an irreducible component of $\Sigma_{k}(G)$ such that $\operatorname{dim}_{\mathbb{C}} V \geq 1$, consider Shd $V$ (not necessarily in $\Sigma_{k}(G)$ ) parallel to $V$ (Shd $V=\rho V$ for some $\rho \in \mathbb{T}_{G}$ ) and such that $\mathbf{1} \in$ Shd $V$. Such a subtorus Shd $V$ will be referred to as the shadow of $V$.

The result and the proof of Theorem 1 provide many obstructions for the quasiprojectivity of a group. We start with a very useful result (see Lemma 5.2).

Lemma 6.4 Let $\xi$ be a non-torsion character such that $H^{1}\left(X ; \mathbb{C}_{\xi}\right) \neq 0$. Then there is a unique maximal orbifold mapping $f: X \rightarrow C_{\varphi}$ such that $\xi=f^{*} \xi_{C}$ and $H^{1}\left(X ; \mathbb{C}_{\xi}\right)=$ $f^{*} H^{1}\left(C_{\varphi} ; \mathbb{C}_{\xi_{C}}\right)$.

Proof Exchanging $\xi$ by $\bar{\xi}$ if necessary we may assume that $H_{\xi}^{\mathcal{O}} \neq 0$. Let us assume that $f_{j}: X \rightarrow C_{j, \varphi}, j=1,2$, satisfies $\xi=f^{*} \xi_{C_{j}}$ and

$$
f_{j}^{*} H^{1}\left(C_{j, \varphi} ; \mathbb{C}_{\xi_{C_{j}}}\right) \subset H^{1}\left(X ; \mathbb{C}_{\xi}\right) \text {. }
$$


One can remove points from $C_{j}$ to ensure $H_{\bar{\xi}_{j}}^{\mathcal{O}} \neq 0$ (replacing $X$ by a dense Zariskiopen set). The foliation argument used in Lemma 5.2 gives a contradiction.

Proposition 6.5 Let $G$ be a quasi-projective group and $V_{1}, V_{2}$ different irreducible components of $\Sigma_{k}(G), k \geq 1$ of positive dimension. Then:

(1) If the intersection $V_{1} \cap V_{2}$ is non-empty, then it consists of isolated torsion points.

(2) Their shadows are either equal or have $\mathbf{1}$ as an isolated intersection point.

(3) If $V_{1}$ is not a component of $\Sigma_{k+1}(G)$ and $p \in V_{1} \cap \Sigma_{k+1}(G)$, then $p$ is a torsion point.

(4) $V_{1}$ is an irreducible component of $\Sigma_{\ell}(G), 1 \leq \ell \leq k$.

Proof Property (4) follows from Lemma 6.4 since it is true for orbifolds. Each irreducible component comes from an orbifold; if two different components come from the same orbifold, they are parallel, hence disjoint. Lemma 6.4 gives (1).

If the underlying Riemann surface of the orbifold giving $V_{j}$ is not $\mathbb{C}^{*}$ or an elliptic curve, then their shadows are in $\Sigma_{1}$, and (2) follows from (1). The general case is proved by Dimca. Finally (3) is an immediate consequence of (1) and Theorem 1.

Remark 6.6 Parts (1) and (2) in Proposition 6.5 can also be found in [29]. Part (3) is proved in [26].

Proposition 6.7 Let $G$ be a quasi-projective group and let $V$ be an irreducible component of $\Sigma_{k}(G), k \geq 1$ of positive dimension $d$. Then:

(1) If $\mathbf{1} \in V$, then $k \leq d-1$. Moreover, one can ensure that $V$ is a component of $\Sigma_{d-e}(G)$, where $e=2$ if $d$ is even and $e=1$ if $d$ is odd.

(2) If $1 \notin V$, then $V$ is a component of $\Sigma_{d}(G)$.

(3) If $1 \notin V$ and $d>2$, then its shadow is an irreducible component of $\Sigma_{1}(G)$.

(4) If $\mathbf{1} \notin V$ and $d=2$, then its shadow is an irreducible component of $\Sigma_{1}(G)$ if and only if its shadow is an irreducible component of $\Sigma_{2}(G)$.

(5) If $\mathbf{1} \notin V$ and $d=1$, then its shadow is not an irreducible component of $\Sigma_{1}(G)$.

Proof This is a direct consequence of Theorem 1 and the properties of the characteristic varieties of orbifolds. 
Remark 6.8 The results (4) and (5) in Proposition 6.7 can be found in [26]. The cases where the shadow is not in the characteristic variety correspond, according to Theorem 1 , to either orbifold pencils over $\mathbb{C}^{*}$ or elliptic pencils.

Proposition 6.9 Let $G$ be a quasi-projective group, and let $V_{1}$ and $V_{2}$ be two distinct irreducible components of $\Sigma_{k}(G)$, resp. $\Sigma_{\ell}(G)$. If $\xi \in V_{1} \cap V_{2}$, then this torsion point satisfies $\xi \in \Sigma_{k+\ell}(G)$.

Proof Let $H_{j} \subset H^{1}\left(X ; \mathbb{C}_{\xi}\right)$ be the subspace obtained by the pull-back of the orbifold giving $V_{j}, j=1,2, \operatorname{dim} H_{1}=k, \operatorname{dim} H_{2}=\ell$. Using the arguments of the proof of Lemma 6.4 we prove $H_{1} \cap H_{2}=\{0\}$.

Remark 6.10 A careful look at the proof of Proposition 6.9 shows stronger consequences. In particular, the hypothesis that the irreducible components $V_{1}$ and $V_{2}$ be distinct can be weakened as follows: $V_{1}$ and $V_{2}$ are $\xi$-distinct as long as the spaces $H_{j} \subset H^{1}\left(X ; \mathbb{C}_{\xi}\right)$ obtained in the proof are different. Analogously, using the arguments of the proof of Lemma 6.4 one obtains that $H_{1} \cap H_{2}=\{0\}$ and hence $\xi \in \Sigma_{k+\ell}(G)$.

This subtle improvement is illustrated in Example 7.2.

\section{Examples}

Example 7.1 Consider the curve $C$ with equation

$$
x y z\left(x^{2}+y^{2}+z^{2}-2 x y-2 x z-2 y z\right)=0 .
$$

The fundamental group $G$ of $X:=\mathbb{P}^{2} \backslash C$ is the Artin group of the triangle with weights $(2,4,4)$; see [5]. The first characteristic variety of $X$ consists of three irreducible components of dimension 1 , intersecting at one character $\xi$ of order 2 . The second characteristic variety equals $\{\xi\}$ and the third one is empty. This illustrates Proposition 6.9.

Example 7.2 Let us consider a curve $C \subset \mathbb{P}^{2}$ that is the dual curve of a smooth cubic and let $X:=\mathbb{P}^{2} \backslash C$. Note that $C$ is a curve of degree 6 with nine ordinary cusps and $\mathbb{T}_{G}$ is the set of sixth roots of unity, where $G:=\pi_{1}(X)$. The fundamental group of this curve $G$ was computed by Zariski [44]. It is not difficult to prove that $\Sigma_{k}(X), k=1,2,3$, equals the set $\left\{\xi_{6}, \bar{\xi}_{6}\right\}$ of points of order $6, \xi_{6}:=\exp (\sqrt{-1} \pi / 3)$. Concretely, let us define a curve $C$ of equation:

$$
f(x, y, z):=x^{6}+y^{6}+z^{6}-2\left(x^{3} y^{3}+x^{3} z^{3}+y^{3} z^{3}\right)=0
$$




\section{Since}

$$
f(x, y, z)=\left(x^{3}+y^{3}-z^{3}\right)^{2}-4(x y)^{3},
$$

the map $[x: y: z] \mapsto\left[\left(x^{3}+y^{3}-z^{3}\right)^{2}:(x y)^{3}\right]$ induces an orbifold rational map $\mathbb{P}^{2} \rightarrow \mathbb{P}_{2,3}^{1}$, for which $C \mapsto[4: 1]$. Therefore it also determines an orbifold map $X \rightarrow \mathbb{C}_{2,3}$, which produces (by pull-back) a non-zero element $\theta_{z} \in H_{\xi_{6}}^{\mathcal{O}} \subset H^{1}\left(X ; \mathbb{C}_{\xi_{6}}\right)$. From the symmetry of the equation one can obtain two other elements $\theta_{y}$ and $\theta_{x}$. Even though $\theta_{x}, \theta_{y}$ and $\theta_{z}$ satisfy the statement of Theorem 5.1, one can easily construct 1 -forms not satisfying it by simply considering a generic linear combination of $\theta_{z}$ and $\theta_{y}$.

Also note that one can find other toric decompositions, like the one defined by

$$
\begin{aligned}
f(x, y, z)=4\left(x^{2}+x z\right. & \left.+x y+z^{2}+z y+y^{2}\right)^{3}-3\left(z^{3}+2 x z^{2}+2 x^{2} z\right. \\
& \left.+x^{3}+2 z^{2} y+2 z y x+2 x^{2} y+2 z y^{2}+2 x y^{2}+y^{3}\right)^{2} .
\end{aligned}
$$

In this case, the forms obtained using these pencils generate $H^{1}\left(X ; \mathbb{C}_{\xi_{6}}\right)$. This example can also be found in [19], where an infinite number of decompositions of type $f h^{6}=$ $g_{3}^{2}+g_{3}^{2}$ are described. Such decompositions are called quasi-toric decompositions and they produce morphisms onto $\mathbb{P}_{(2,3,6)}^{1}$.

This illustrates Remark 6.10, since for instance $V_{1}=V_{2}=\left\{\xi_{6}\right\} \subset \Sigma_{1}(X)$ can be chosen such that $V_{1}$ (resp. $V_{2}$ ) is associated with the orbifold map coming from (7-1) (resp. (7-2)). This automatically implies that $\xi_{6} \in \Sigma_{2}(X)$.

Note that $\mathbb{T}_{\Pi}=\bigcup_{\lambda} \mathbb{T}_{\Pi}^{\lambda}$, where $\Pi=\pi^{\text {orb }}\left(\mathbb{C}_{2,3}\right)=\mathbb{F}_{2,3}^{0}, \lambda=\left(\lambda_{1}, \lambda_{2}\right) \in C_{2} \times C_{3}$, and $\mathbb{T}_{\Pi}^{(1,1)}=\{\mathbf{1}\}$. According to Proposition 2.10,

$$
\Sigma_{1}(\Pi)=\mathbb{T}_{\Pi}^{\left(-1, \xi_{3}\right)} \cup \mathbb{T}_{\Pi}^{\left(-1, \bar{\xi}_{3}\right)}=\left\{\xi_{6}, \bar{\xi}_{6}\right\} \quad \text { and } \quad \Sigma_{2}(\Pi)=\varnothing
$$

$\left(\xi_{n}:=\exp (2 \pi \sqrt{-1} / n)\right)$.

Summarizing, $\xi_{6}$ as an element of $\Sigma_{1}$ is of pencil type. However, if we consider $\xi_{6}$ as an element of $\Sigma_{2}$, then it is not of pencil type.

Example 7.3 The affine Degtyarev curve $C \subset \mathbb{C}^{2}$ in [3, Section 3] provides an example of a space $X=\mathbb{C}^{2} \backslash C$ such that $\Sigma_{1}(X)=\left\{\xi \mid \xi^{4}-\xi^{3}+\xi^{2}-\xi+1=0\right\} \cup\{\mathbf{1}\}$, but $\xi$ is not of pencil type. Note that in this case $\xi$ is a torsion character and both $b_{1}\left(X^{\xi}\right)=b_{1}(\bar{X})=0$, which is necessary by Remark 5.7.

Example 7.4 Consider the following arrangement (cf Barthel, Hirzebruch and Höfer [6]) given by equations $C_{n}:=\left(y^{n}-x^{n}\right)\left(y^{n}-z^{n}\right)\left(z^{n}-x^{n}\right), n \geq 2$, where

$$
\ell_{1+3 k}:=y-\xi_{n}^{k} z, \quad \ell_{2+3 k}:=z-\xi_{n}^{k} x, \quad \ell_{3+3 k}:=y-\xi_{n}^{k} x .
$$


This arrangement can be seen as the Kummer covering $[x: y: z] \mapsto\left[x^{n}: y^{n}: z^{n}\right]$ of a Ceva arrangement ramified along $\{x y z=0\}$. It has been considered by Cohen in [20], by Cohen, Denham and Suciu in [21], and by Dimca in [25], where mainly the components in $\Sigma_{1}$ have been accounted for. Here we interpret the essential components in $\Sigma_{k}$ for $k>1$.

Note the following pencils associated with the arrangement $C_{n}$ :

$$
F_{\alpha, \beta}=\alpha f_{1}+\beta f_{2}:=\alpha x^{n} \prod_{k=1}^{n} \ell_{1+3 k}+\beta y^{n} \prod_{k=1}^{n} \ell_{2+3 k}=\alpha x^{n}\left(y^{n}-z^{n}\right)+\beta y^{n}\left(z^{n}-x^{n}\right),
$$

where

$$
f_{1}+f_{2}=z^{n}\left(y^{n}-x^{n}\right)=z^{n} \prod_{k=1}^{n} \ell_{3+3 k}
$$

and

$$
G_{\alpha, \beta}=\alpha g_{1}+\beta g_{2}:=\alpha \prod_{k=1}^{n} \ell_{1+3 k}+\beta \prod_{k=1}^{n} \ell_{2+3 k}=\alpha\left(y^{n}-z^{n}\right)+\beta\left(z^{n}-x^{n}\right),
$$

where

$$
g_{1}+g_{2}=\left(y^{n}-x^{n}\right)=\prod_{k=1}^{n} \ell_{3+3 k} .
$$

The rational maps $f_{i}: \mathbb{P}^{2} \rightarrow \mathbb{P}^{1}, i=1,2$, defined by $f_{1}([x: y: z]):=\left[f_{1}: f_{2}\right]$, $f_{2}([x: y: z]):=\left[g_{1}: g_{2}\right]$, are such that $\{x y z=0\} \cup C_{n}=f_{1}^{-1}(\{[0: 1],[1: 0],[1:-1]\})$ and $C_{n}=f_{2}^{-1}(\{[0: 1],[1: 0],[1:-1]\})$. Moreover, after resolving the base points of the rational maps one obtains two morphisms:

$$
\begin{aligned}
& \tilde{f}_{1}: X_{n} \rightarrow \Omega_{1}:=\mathbb{P}_{n, n, n}^{1}, \\
& \tilde{f}_{2}: X_{n} \rightarrow \Omega_{2}:=\mathbb{P}^{1} \backslash\{[0: 1],[1: 0],[1:-1]\},
\end{aligned}
$$

where $X_{n}=\mathbb{P}^{2} \backslash C_{n}$. By Proposition 2.11,

$$
\Sigma_{1}\left(\Omega_{1}\right)=\left\{\lambda=\left(\xi_{n}^{i}, \xi_{n}^{j}, \xi_{n}^{-(i+j)}\right) \mid \ell(\lambda) \geq 3\right\} \supset \Sigma_{2}\left(\Omega_{1}\right)=\varnothing,
$$

$\Sigma_{1}\left(\Omega_{2}\right)=\mathbb{T}_{\mathbb{F}^{2}} \supset \Sigma_{2}\left(\Omega_{2}\right)=\varnothing$. The injection $\tilde{f}_{1}^{*}\left(\Sigma_{1}\left(\Omega_{1}\right)\right) \subset \Sigma_{1}\left(X_{n}\right)$ produces a zero-dimensional embedded component inside the one-dimensional component

$$
\tilde{f}_{2}^{*}\left(\Sigma_{2}\left(\Omega_{2}\right)\right) \subset \Sigma_{2}\left(X_{n}\right)
$$

(except for $n=2)$. Using Proposition 6.9 one can deduce that in fact $\tilde{f}_{1}^{*}\left(\Sigma_{1}\left(\Omega_{1}\right)\right) \subset$ $\Sigma_{2}(X)$. This was already pointed out in [25], but here we give a different approach. 


\section{References}

[1] D Arapura, Geometry of cohomology support loci for local systems, I, J. Algebraic Geom. 6 (1997) 563-597 MR1487227

[2] E Artal Bartolo, J Carmona Ruber, J I Cogolludo-Agustín, Essential coordinate components of characteristic varieties, Math. Proc. Cambridge Philos. Soc. 136 (2004) 287-299 MR2040575

[3] E Artal Bartolo, J I Cogolludo-Agustín, On the connection between fundamental groups and pencils with multiple fibers, J. Singul. 2 (2010) 1-18 MR2763015

[4] E Artal Bartolo, J I Cogolludo-Agustín, A Libgober, Depth of cohomology support loci for quasi-projective varieties via orbifold pencils arXiv:1203.2663v1

[5] E Artal Bartolo, J I Cogolludo-Agustín, D Matei, Quasi-projectivity, Artin-Tits groups, and pencil maps, from: "Topology of algebraic varieties and singularities", (J I Cogolludo-Agustín, E Hironaka, editors), Contemp. Math. 538, Amer. Math. Soc. (2011) 113-136 MR2777818

[6] G Barthel, F Hirzebruch, T Höfer, Geradenkonfigurationen und Algebraische Flächen, Aspects of Mathematics 4, Friedr. Vieweg \& Sohn, Braunschweig (1987) MR912097

[7] I Bauer, Irrational pencils on non-compact algebraic manifolds, Internat. J. Math. 8 (1997) 441-450 MR1460895

[8] A Beauville, Annulation du $H^{1}$ pour les fibrés en droites plats, from: "Complex algebraic varieties”, (K Hulek, T Peternell, M Schneider, F-O Schreyer, editors), Lecture Notes in Math. 1507, Springer, Berlin (1992) 1-15 MR1178716

[9] R Bieri, W D Neumann, R Strebel, A geometric invariant of discrete groups, Invent. Math. 90 (1987) 451-477 MR914846

[10] F Bruhat, J Tits, Groupes réductifs sur un corps local, Inst. Hautes Études Sci. Publ. Math. (1972) 5-251 MR0327923

[11] N Budur, Unitary local systems, multiplier ideals, and polynomial periodicity of Hodge numbers, Adv. Math. 221 (2009) 217-250 MR2509325

[12] F Campana, Ensembles de Green-Lazarsfeld et quotients résolubles des groupes de Kähler, J. Algebraic Geom. 10 (2001) 599-622 MR1838973

[13] F Campana, Orbifoldes géométriques spéciales et classification biméromorphe des variétés kählériennes compactes, J. Inst. Math. Jussieu 10 (2011) 809-934 MR2831280

[14] F Campana, Quotients résolubles ou nilpotents des groupes de Kähler orbifoldes, Manuscripta Math. 135 (2011) 117-150 MR2783390

[15] F Campana, Special orbifolds and birational classification: a survey, from: "Classification of algebraic varieties", (C Faber, G van der Geer, E Looijenga, editors), EMS Ser. Congr. Rep., Eur. Math. Soc., Zürich (2011) 123-170 MR2779470 
[16] F Catanese, Moduli and classification of irregular Kaehler manifolds (and algebraic varieties) with Albanese general type fibrations, Invent. Math. 104 (1991) 263-289 MR1098610

[17] F Catanese, J Keum, K Oguiso, Some remarks on the universal cover of an open K3 surface, Math. Ann. 325 (2003) 279-286 MR1962049

[18] J I Cogolludo-Agustín, Topological invariants of the complement to arrangements of rational plane curves, Mem. Amer. Math. Soc. 159 (2002) xiv+75 MR1921584

[19] J I Cogolludo-Agustín, A Libgober, Mordell-Weil groups of elliptic threefolds and the Alexander module of plane curves, to appear in Crelle's Journal arXiv:1008.2018

[20] D C Cohen, Triples of arrangements and local systems, Proc. Amer. Math. Soc. 130 (2002) 3025-3031 MR1908926

[21] D C Cohen, G Denham, A I Suciu, Torsion in Milnor fiber homology, Algebr. Geom. Topol. 3 (2003) 511-535 MR1997327

[22] K Corlette, C Simpson, On the classification of rank-two representations of quasiprojective fundamental groups, Compos. Math. 144 (2008) 1271-1331 MR2457528

[23] P Deligne, Équations différentielles à points singuliers réguliers, Lecture Notes in Mathematics 163, Springer, Berlin (1970) MR0417174

[24] T Delzant, Trees, valuations and the Green-Lazarsfeld set, Geom. Funct. Anal. 18 (2008) 1236-1250 MR2465689

[25] A Dimca, Characteristic varieties and constructible sheaves, Atti Accad. Naz. Lincei Cl. Sci. Fis. Mat. Natur. Rend. Lincei Mat. Appl. 18 (2007) 365-389 MR2349994

[26] A Dimca, On the irreducible components of characteristic varieties, An. Ştiinţ. Univ. “Ovidius” Constanţa Ser. Mat. 15 (2007) 67-73 MR2396745

[27] A Dimca, On admissible rank one local systems, J. Algebra 321 (2009) 3145-3157 MR2510044

[28] A Dimca, L Maxim, Multivariable Alexander invariants of hypersurface complements, Trans. Amer. Math. Soc. 359 (2007) 3505-3528 MR2299465

[29] A Dimca, Ş Papadima, A I Suciu, Alexander polynomials: essential variables and multiplicities, Int. Math. Res. Not. 2008 (2008) Art. ID rnm119, 36 MR2416998

[30] A Dimca, Ş Papadima, A I Suciu, Topology and geometry of cohomology jump loci, Duke Math. J. 148 (2009) 405-457 MR2527322

[31] R H Fox, Free differential calculus, V: the Alexander matrices re-examined, Ann. of Math. 71 (1960) 408-422 MR0111781

[32] M Green, R Lazarsfeld, Deformation theory, generic vanishing theorems, and some conjectures of Enriques, Catanese and Beauville, Invent. Math. 90 (1987) 389-407 MR910207

[33] H A Hamm, Lefschetz theorems for singular varieties, from: "Singularities, Part 1", (P Orlik, editor), Proc. Sympos. Pure Math. 40, Amer. Math. Soc., Providence, RI (1983) 547-557 MR713091 
[34] E Hironaka, Alexander stratifications of character varieties, Ann. Inst. Fourier (Grenoble) 47 (1997) 555-583 MR1450425

[35] G Levitt, R-trees and the Bieri-Neumann-Strebel invariant, Publ. Mat. 38 (1994) 195-202 MR1291961

[36] A Libgober, Characteristic varieties of algebraic curves, from: "Applications of algebraic geometry to coding theory, physics and computation”, (C Ciliberto, F Hirzebruch, R Miranda, M Teicher, editors), NATO Sci. Ser. II Math. Phys. Chem. 36, Kluwer Acad. Publ., Dordrecht (2001) 215-254 MR1866902

[37] A Libgober, Non vanishing loci of Hodge numbers of local systems, Manuscripta Math. 128 (2009) 1-31 MR2470184

[38] M V Nori, Zariski's conjecture and related problems, Ann. Sci. École Norm. Sup. 16 (1983) 305-344 MR732347

[39] F Serrano, Multiple fibres of a morphism, Comment. Math. Helv. 65 (1990) 287-298 MR1057245

[40] C Simpson, Lefschetz theorems for the integral leaves of a holomorphic one-form, Compositio Math. 87 (1993) 99-113 MR1219454

[41] C Simpson, Subspaces of moduli spaces of rank one local systems, Ann. Sci. École Norm. Sup. 26 (1993) 361-401 MR1222278

[42] Y T Siu, Strong rigidity for Kähler manifolds and the construction of bounded holomorphic functions, from: "Discrete groups in geometry and analysis", (R Howe, editor), Progr. Math. 67, Birkhäuser, Boston, MA (1987) 124-151 MR900825

[43] K Timmerscheidt, Mixed Hodge theory for unitary local systems, J. Reine Angew. Math. 379 (1987) 152-171 MR903638

[44] O Zariski, The topological discriminant group of a Riemann surface of genus $p$, Amer. J. Math. 59 (1937) 335-358 MR1507244

EA, JC: Departamento de Matemáticas, IUMA, Facultad de Ciencias, Universidad de Zaragoza

c/ Pedro Cerbuna 12, E-50009 Zaragoza, Spain

DM: Institute of Mathematics of the Romanian Academy

P.O. Box 1-764, 014700 Bucharest, Romania

artal@unizar.es, jicogo@unizar.es, Daniel.Matei@imar.ro

http://riemann.unizar.es/geotop/WebGeoTo/Profes/eartal/, http://riemann.unizar.es/ jicogo/, http://www.imar.ro/ dmatei

Proposed: Lothar Göttsche

Seconded: Walter Neumann, Jim Bryan
Received: 3 May 2012 Accepted: 22 September 2012 